\title{
Cramér-Rao Bounds for Blind Multichannel Estimation
}

\author{
Elisabeth de Carvalho \\ Aalborg University \\ Dept. of Electronic Systems \\ Selma LagerlØfs Vej 312 \\ 9220 Aalborg, DENMARK \\ Tel: +459940 7528 \\ Email: edc@es.aau.dk
}

\author{
and
}

\author{
Dirk T.M. Slock \\ EURECOM \\ Communication Systems Dept. \\ 450 Route des Chappes \\ 06410 BIOT Sophia Antipolis, FRANCE \\ Tel: +33493002606 \\ email: slock@eurecom.fr
}

Written December 1999

\begin{abstract}
In some estimation problems, not all the parameters can be identified, which results in singularity of the Fisher Information Matrix (FIM). The Cramér-Rao Bound (CRB), which is the inverse of the FIM, is then not defined. To regularize the estimation problem, one can impose constraints on the parameters and derive the corresponding CRBs. The correspondence between local identifiability and FIM regularity is studied here. Furthermore the number of FIM singularities is shown to be equal to the number of independent constraints necessary to have a well-defined constrained CRB and local identifiability. In general, many sets of constraints can render the parameters identifiable, giving different values for the $\mathrm{CRB}$, that are not always relevant. When the constraints can be chosen, we propose a constrained CRB, the pseudo-inverse of the FIM, which gives, for a minimum number of constraints, the lowest bound on the mean squared estimation error. These results are applied to two approaches to blind FIR multichannel estimation which allow identification of the channel up to a scale or phase factor. These two approaches correspond to deterministic and Gaussian models for the unknown channel inputs. The singularities of the FIMs and local identifiability are studied and the corresponding constrained CRBs are derived and interpreted.
\end{abstract}

Keywords: Fisher Information Matrix, Cramér-Rao Bound, constrained parameter estimation, blind channel identification.

$\dagger$ The work of Elisabeth de Carvalho was supported by Laboratoires d'Electronique Philips under contract Cifre 297/95. The work of Dirk Slock was supported in part by Laboratoires d'Electronique Philips under contract LEP 95FAR008. 


\section{Introduction}

The Cramér-Rao Bound (CRB) is a powerful tool in estimation theory as it gives a performance lower bound for parameter estimation problems. It is computed as the inverse of the Fisher Information Matrix (FIM). When the parameters cannot be completely identified, the FIM is singular, and the classical CRB results cannot be applied directly.

The main underlying motivation of this work is the study of the performance of certain blind channel estimation problems where the parameters can indeed be identified only up to scale or phase factor [1]. Blind estimation is done under certain parameter constraints to regularize the problem. The performance of blind methods is not correctly evaluated in general or remains somewhat vague. A constraint often used [2] is to consider one coefficient of the channel as known (which is sufficient to render the estimation problem regular): the resulting performance and its bound depend on the choice of this coefficient and appear arbitrary. One of the contributions of this work will be to give a less arbitrary bound and the corresponding set of constraints. Another motivation comes from other work [1, 3] by the authors in which blind and semi-blind methods are compared through the CRBs. To get a meaningful comparison, semi-blind and blind CRBs have to be computed under the same constraints. For that purpose, this study, which is valid for the regular or the non regular estimation problem, was then necessary.

The first part of this paper focuses first on the FIMs and especially the correspondence, for a Gaussian data distribution, between FIM regularity and parameter identifiability, defined in terms of a probability density function. For the blind channel estimation application considered here, FIM regularity and local identifiability are equivalent. In a second step, we study the CRBs for estimation under parameter constraints. A similar study was done in 4 for the case where the unconstrained problem is identifiable, i.e. the FIM is regular. We adapt here the results to the case where the unconstrained problem leads to nonidentifiability, i.e. the FIM is singular. We furthermore outline the correspondence between the number and characteristics of FIM singularities and the number and characteristics of independent constraints needed in order to regularize the estimation problem and to be able to define the constrained CRB. In a last step, assuming that we can choose the set of constraints, we propose a particular CRB for the case of an unidentifiable unconstrained estimation problem: this CRB is the Moore-Penrose pseudo-inverse of the FIM. It corresponds to a minimum number of particular constraints and gives the lowest bound on the mean square estimation error, i.e. $\operatorname{tr}(C R B)$.

The second part of this paper focuses on two classes of blind FIR multichannel estimation problems corresponding to two different models for the input symbols. The deterministic model, which exploits no statistical information on the input symbols, takes the input symbols to be deterministic quantities whereas in the Gaussian model we consider them to be Gaussian random variables to exploit their second-order statistics. The deterministic model leads to the class of methods directly based on the structure of the received signal; the Gaussian approach includes methods based on the second-order moments of the data, like certain prediction approaches [5] or the covariance matching method [6]. We refer to [1] for a more complete description of the two models. The deterministic methods can identify the channel up to a scale factor only and the Gaussian methods up to a phase factor, resulting in singularities of the FIM.

The FIM singularities are studied in both cases and hence also the conditions for local identifiability. The deterministic model cannot identify the zeros of a multichannel whereas the Gaussian model can, under certain conditions given here. Throughout the paper, we distinguish between the real and complex parameter cases since they lead to different FIMs, with different singularities, and require different regularization constraints. The blind deterministic CRB is computed under the commonly used norm constraint which imposes the norm of the channel to be constant. This constraint is sufficient to regularize the problem when the channel is real, but not when it is complex in which case an additional constraint is required to adjust the phase of the channel. This constraint is chosen so that the resulting constrained CRB is the Moore-Penrose pseudo-inverse of the FIM and corresponds to a minimal constrained CRB. When the channel is real the Gaussian FIM is regular, when it is complex however, the FIM is singular: a constraint on the phase is necessary as in the deterministic case and the constrained CRB is again the pseudo-inverse of the FIM. In some examples, we illustrate furthermore the variation of the constrained CRB according to the constraint chosen and especially compare the pseudo-inverse of the FIM to the constrained CRB corresponding to the assumed knowledge of one coefficient of the channel.

Some notations and acronyms that will be used in the paper are:

$$
(.)^{*},(.)^{T},(.)^{H} \quad \text { conjugate, transpose, conjugate transpose }
$$




$\begin{array}{ll}(.)^{+} & \text {Moore-Penrose pseudo-inverse } \\ \operatorname{tr}(A), \operatorname{det}(A) & \text { trace and determinant of matrix } A \\ \operatorname{vec}(A) & {\left[A_{i, 1}^{T} A_{i, 2}^{T} \cdots A_{i, n}^{T}\right]^{T}} \\ \otimes & \text { Kronecker product } \\ \hat{\theta}, \theta^{o} & \text { estimate of parameter } \theta, \text { true value of parameter } \theta \\ \mathrm{E}_{X} & \text { mathematical expectation w.r.t. the random quantity } X \\ \operatorname{Re}(.), \operatorname{Im}(.) & \text { real and imaginary part } \\ I & \text { identity matrix with adequate dimension } \\ \text { w.r.t. } & \text { with respect to }\end{array}$

\section{CRBs for Real and Complex Parameters}

We assume here the FIMs to be regular.

\subsection{CRBs for Real Parameters}

Let $\theta$ be a deterministic real parameter vector and $f(\boldsymbol{Y} \mid \theta)$ the probability density function of the vector of observations $\boldsymbol{Y}$. The FIM associated with $\theta$ is:

$$
\mathcal{J}_{\theta \theta}=\mathrm{E}_{Y \mid \theta}\left(\frac{\partial \ln f(\boldsymbol{Y} \mid \theta)}{\partial \theta}\right)\left(\frac{\partial \ln f(\boldsymbol{Y} \mid \theta)}{\partial \theta}\right)^{T} .
$$

Let $\hat{\theta}$ be an unbiased estimate of $\theta$ and $\tilde{\theta}=\hat{\theta}-\theta$ the estimation error. Hence $\mathrm{E} \tilde{\theta}=0$ and $\mathcal{C}_{\tilde{\theta} \tilde{\theta}}=\mathrm{E} \tilde{\theta} \tilde{\theta}^{T}$ is the error covariance matrix. When $\mathcal{J}_{\theta \theta}$ is nonsingular and under certain regularity conditions [7], $\mathcal{J}_{\theta \theta}^{-1}$ is the Cramér-Rao Bound and:

$$
\mathcal{C}_{\tilde{\theta} \tilde{\theta}} \geq C R B=\mathcal{J}_{\theta \theta}^{-1}
$$

Equality is achieved if and only if:

$$
\hat{\theta}-\theta=\mathcal{J}_{\theta \theta}^{-1} \frac{\partial \ln f(\boldsymbol{Y} \mid \theta)}{\partial \theta}
$$

\subsection{CRB for Complex Parameters, Complex CRB.}

When $\theta$ is a complex deterministic parameter, the previous results can be applied to $\theta_{R}=\left[\operatorname{Re}^{T}(\theta) \operatorname{Im}^{T}(\theta)\right]^{T}$ and $\boldsymbol{Y}_{R}=\left[\operatorname{Re}^{T}(\boldsymbol{Y}) \operatorname{Im}^{T}(\boldsymbol{Y})\right]^{T}$, the associated real parameters and real observations.

It is however possible to define the FIM for $\theta_{R}$ w.r.t. complex FIM-like matrices. Let $J_{\varphi \psi}$ be defined as:

$$
J_{\varphi \psi}=\mathrm{E}_{Y \mid \theta}\left(\frac{\partial \ln f(\boldsymbol{Y} \mid \theta)}{\partial \varphi^{*}}\right)\left(\frac{\partial \ln f(\boldsymbol{Y} \mid \theta)}{\partial \psi^{*}}\right)^{H}
$$

where $f(\boldsymbol{Y} \mid \theta)=f\left(\boldsymbol{Y}_{R} \mid \theta\right)=f\left(\boldsymbol{Y}_{R} \mid \theta_{R}\right)$. Derivation w.r.t. the complex vector $\theta=\alpha+j \beta$ is defined as: $\frac{\partial}{\partial \theta}=\frac{1}{2}\left(\frac{\partial}{\partial \alpha}-j \frac{\partial}{\partial \beta}\right)$ (see [7] for some properties of complex derivation). Remark that we denote real and complex FIMs by $\mathcal{J}$ and $J$ respectively.

The parametrization in $(\operatorname{Re}(\theta), \operatorname{Im}(\theta))$ is equivalent to a parametrization in terms of $\left(\theta, \theta^{*}\right)$ via:

$$
\theta_{R}=\left[\begin{array}{c}
\operatorname{Re}(\theta) \\
\operatorname{Im}(\theta)
\end{array}\right]=\mathcal{M}\left[\begin{array}{c}
\theta \\
\theta^{*}
\end{array}\right], \quad \mathcal{M}=\frac{1}{2}\left[\begin{array}{cc}
I & I \\
-j I & j I
\end{array}\right]
$$

where $\mathcal{M}$ is non-singular. Knowing that $J_{\theta \theta}=J_{\theta^{*} \theta^{*}}^{*}$ and $J_{\theta \theta^{*}}=J_{\theta^{*} \theta}^{*}$ (true here as $f_{\boldsymbol{Y} \mid \theta}\left(\theta, \theta^{*}\right)=$ $\left.f_{\boldsymbol{Y} \mid \theta}\left(\theta^{*}, \theta\right)\right)$, equation (5) implies:

$$
\mathcal{J}_{\theta_{R} \theta_{R}}=\mathcal{M}\left[\begin{array}{cc}
J_{\theta \theta} & J_{\theta \theta^{*}} \\
J_{\theta \theta^{*}}^{*} & J_{\theta \theta}^{*}
\end{array}\right] \mathcal{M}^{H}
$$

$\mathcal{J}_{\theta_{R} \theta_{R}}$ can be determined from $J_{\theta \theta}$ and $J_{\theta \theta^{*}}$ as follows:

$$
\mathcal{J}_{\theta_{R} \theta_{R}}=2\left[\begin{array}{rr}
\operatorname{Re}\left(J_{\theta \theta}\right) & -\operatorname{Im}\left(J_{\theta \theta}\right) \\
\operatorname{Im}\left(J_{\theta \theta}\right) & \operatorname{Re}\left(J_{\theta \theta}\right)
\end{array}\right]+2\left[\begin{array}{rr}
\operatorname{Re}\left(J_{\theta \theta^{*}}\right) & \operatorname{Im}\left(J_{\theta \theta^{*}}\right) \\
\operatorname{Im}\left(J_{\theta \theta^{*}}\right) & -\operatorname{Re}\left(J_{\theta \theta^{*}}\right)
\end{array}\right] .
$$


To quantify the estimation quality, the quantity of interest will be $\operatorname{tr}\left(C R B_{R}\right)$, i.e. the lower bound on the mean squared estimation error, which can be expressed directly in terms of the quantities $J_{\theta \theta}$ and $J_{\theta \theta^{*}}$. Since $\mathcal{M} \mathcal{M}^{H}=\frac{1}{2} I, \sqrt{6}$ implies:

$$
\mathcal{J}_{\theta_{R} \theta_{R}}^{-1}=4 \mathcal{M}\left[\begin{array}{cc}
J_{\theta \theta} & J_{\theta \theta^{*}} \\
J_{\theta \theta^{*}}^{*} & J_{\theta \theta}^{*}
\end{array}\right]^{-1} \mathcal{M}^{H}
$$

Then:

$$
\operatorname{tr}\left(C R B_{R}\right)=\operatorname{tr}\left(\mathcal{J}_{\theta_{R} \theta_{R}}^{-1}\right)=4 \operatorname{tr}\left(J_{\theta \theta}-J_{\theta \theta^{*}} J_{\theta \theta}^{-*} J_{\theta \theta^{*}}^{*}\right)^{-1}
$$

Theorem 1 When $J_{\theta \theta^{*}}=0, \mathcal{J}_{\theta_{R} \theta_{R}}$ is completely determined by $J_{\theta \theta}$. In that case, $J_{\theta \theta}$ can be considered as the complex FIM and the corresponding complex CRB is such that:

$$
C_{\tilde{\theta} \tilde{\theta}}=E \tilde{\theta} \tilde{\theta}^{H} \geq C R B=J_{\theta \theta}^{-1}
$$

If $J_{\theta \theta^{*}} \neq 0, J_{\theta \theta}^{-1}$ is also a lower bound on $C_{\tilde{\theta} \tilde{\theta}}$, but not as tight as the (real) $C R B, C R B_{R}$.

In that case $\left(J_{\theta \theta^{*}}=0\right)$, a single complex singularity of the complex FIM $J_{\theta \theta}$ corresponds to two real singularities since if $\theta_{s}$ is a singular vector, then so is $j \theta_{s}$.

\section{CRBs for a Gaussian Data Distribution}

\subsection{Real Parameters}

The CRB for a Gaussian data distribution:

$$
\begin{array}{ll}
\boldsymbol{Y} \sim \mathcal{N}\left(m_{Y}(\theta), C_{Y Y}(\theta)\right), \quad m_{Y}(\theta)=\mathrm{E}_{Y \mid \theta}(\boldsymbol{Y}) \\
& C_{Y Y}(\theta)=\mathrm{E}_{Y \mid \theta}\left(\boldsymbol{Y}-m_{Y}(\theta)\right)\left(\boldsymbol{Y}-m_{Y}(\theta)\right)^{H}
\end{array}
$$

is [7:

$$
\mathcal{J}_{\theta \theta}(i, j)=\left(\frac{\partial m_{Y}^{T}}{\partial \theta_{i}}\right) C_{Y Y}^{-1}\left(\frac{\partial m_{Y}^{T}}{\partial \theta_{j}}\right)^{T}+\frac{1}{2} \operatorname{tr}\left\{C_{Y Y}^{-1}\left(\frac{\partial C_{Y Y}}{\partial \theta_{i}}\right) C_{Y Y}^{-1}\left(\frac{\partial C_{Y Y}}{\partial \theta_{j}}\right)\right\},
$$

where, to simplify, the mean and the covariance matrix are denoted $m_{Y}$ and $C_{Y Y}$.

The FIM can also be expressed in a closed form. Let's define $\phi$ to be a vector comprising the elements of the mean and covariance of the data as:

$$
\phi=\left[\begin{array}{c}
m_{Y} \\
\operatorname{vec}\left\{C_{Y Y}\right\}
\end{array}\right] .
$$

Using the properties: $\operatorname{tr}\{A B\}=\operatorname{vec}^{T}\left\{A^{T}\right\} \operatorname{vec}\{B\}$ and $\operatorname{vec}\{A B C\}=\left(C^{T} \otimes A\right) \operatorname{vec}\{B\}$, we find:

$$
F I M=\left(\frac{\partial m_{Y}^{T}}{\partial \theta}\right) C_{Y Y}^{-1}\left(\frac{\partial m_{Y}^{T}}{\partial \theta}\right)^{T}+\left(\frac{\partial \operatorname{vec}^{T}\left\{C_{Y Y}\right\}}{\partial \theta}\right)\left(C_{Y Y}^{-T} \otimes C_{Y Y}^{-1}\right)\left(\frac{\partial \operatorname{vec}^{T}\left\{C_{Y Y}\right\}}{\partial \theta}\right)^{T}
$$

or

$$
J_{\theta \theta}=\left(\frac{\partial \phi^{T}}{\partial \theta}\right)\left[\begin{array}{cc}
C_{Y Y}^{-1} & 0 \\
0 & C_{Y Y}^{-T} \otimes C_{Y Y}^{-1}
\end{array}\right]\left(\frac{\partial \phi^{T}}{\partial \theta}\right)^{T} .
$$

From this expression, the following theorem, also given in [8], is deduced:

Theorem 2 The FIM for a Gaussian data distribution is regular if and only if $\left(\frac{\partial \phi^{T}}{\partial \theta}\right)$ has full row rank. 


\subsection{Complex Parameters}

In a properly formulated blind channel estimation problem, $\boldsymbol{Y}$ and $\theta$ are simultaneously real or complex. If $\boldsymbol{Y}$ is complex, we shall assume it is circular, i.e. $\mathrm{E} \boldsymbol{Y} \boldsymbol{Y}^{T}=0$. In that case, it is possible to define a complex Gaussian conditional probability density function for $\boldsymbol{Y}$ :

$$
f(\boldsymbol{Y} \mid \theta)=\frac{1}{\pi^{M m} \operatorname{det} C_{Y Y}(\theta)} \exp \left[-\left(\boldsymbol{Y}-m_{Y}(\theta)\right)^{H} C_{Y Y}^{-1}(\theta)\left(\boldsymbol{Y}-m_{Y}(\theta)\right)\right]
$$

where $m_{Y}(\theta)=\mathrm{E}_{Y \mid \theta}(\boldsymbol{Y})$ and $C_{Y Y}(\theta)=\mathrm{E}_{Y \mid \theta}\left(\boldsymbol{Y}-m_{Y}(\theta)\right)\left(\boldsymbol{Y}-m_{Y}(\theta)\right)^{H}$. Based on the complex probability density function $f(\boldsymbol{Y} \mid \theta)$, the computation of the complex FIMs $J_{\theta \theta}$ and $J_{\theta \theta^{*}}$ gives (straightforward extension of [7]):

$$
\left\{\begin{array}{l}
J_{\theta \theta}(i, j)=\left(\frac{\partial m_{Y}^{H}}{\partial \theta_{i}^{*}}\right) C_{Y Y}^{-1}\left(\frac{\partial m_{Y}^{H}}{\partial \theta_{j}^{*}}\right)^{H}+\operatorname{tr}\left\{C_{Y Y}^{-1}\left(\frac{\partial C_{Y Y}}{\partial \theta_{i}^{*}}\right) C_{Y Y}^{-1}\left(\frac{\partial C_{Y Y}}{\partial \theta_{j}^{*}}\right)\right\} \\
J_{\theta \theta^{*}}(i, j)=\left(\frac{\partial m_{Y}^{H}}{\partial \theta_{i}^{*}}\right) C_{Y Y}^{-1}\left(\frac{\partial m_{Y}^{H}}{\partial \theta_{j}^{*}}\right)^{T}+\operatorname{tr}\left\{C_{Y Y}^{-1}\left(\frac{\partial C_{Y Y}}{\partial \theta_{i}^{*}}\right) C_{Y Y}^{-1}\left(\frac{\partial C_{Y Y}}{\partial \theta_{j}^{*}}\right)\right\} .
\end{array}\right.
$$

Proceeding as in the real case, the FIM for $\theta_{R}$ becomes:

$$
\mathcal{J}_{\theta_{R} \theta_{R}}=2 \mathcal{M}\left[\begin{array}{c}
\frac{\partial \phi^{T}}{\partial \theta^{*}} \\
\frac{\partial \phi^{T}}{\partial \theta}
\end{array}\right]\left(I_{2} \otimes\left[\begin{array}{cc}
C_{Y Y}^{-1} & 0 \\
0 & C_{Y Y}^{-T} \otimes C_{Y Y}^{-1}
\end{array}\right]\right)\left[\begin{array}{c}
\frac{\partial \phi^{T}}{\partial \theta^{*}} \\
\frac{\partial \phi^{T}}{\partial \theta}
\end{array}\right]^{H} \mathcal{M}^{H}
$$

Theorem 3 The FIM for a complex Gaussian data distribution is regular if and only if $\left[\begin{array}{c}\frac{\partial \phi^{T}}{\partial \theta^{*}} \\ \frac{\partial \phi^{T}}{\partial \theta}\end{array}\right] \begin{gathered}h a s \\ \text { h }\end{gathered}$ full row rank.

\section{Correspondence between Identifiability and FIM Regularity for a Gaussian Data Distribution}

\subsection{Regular Estimation}

We consider identifiability as defined in [9, 1]. In the regular case, $\theta$ is said identifiable if:

$$
\forall \boldsymbol{Y}, \quad f(\boldsymbol{Y} \mid \theta)=f\left(\boldsymbol{Y} \mid \theta^{\prime}\right) \Rightarrow \theta=\theta^{\prime} .
$$

When the observations have a normal distribution, $\boldsymbol{Y} \sim \mathcal{N}\left(m_{Y}(\theta), C_{Y Y}(\theta)\right)$, identifiability is based on the mean and covariance: $\theta$ is said identifiable if:

$$
m_{Y}(\theta)=m_{Y}\left(\theta^{\prime}\right) \text { and } C_{Y Y}(\theta)=C_{Y Y}\left(\theta^{\prime}\right) \quad \Rightarrow \quad \theta=\theta^{\prime} .
$$

(the mapping: $\theta \rightarrow\left\{m_{Y}, C_{Y Y}\right\}$ is invertible). We have local identifiability at $\theta$ if identifiability holds for $\theta^{\prime}$ being restricted to some open neighborhood of $\theta$. In the Gaussian distribution case, there is a correspondence between FIM regularity and local identifiability.

Theorem 4 If $\theta$ is not locally identifiable at $\theta$, then the FIM is singular at $\theta$ [8].

If a parameter can only be identified up to a continuous ambiguity, for example a scale factor, it cannot be locally identifiable and the corresponding FIM is singular. However, when the parameter is identifiable up to a discrete ambiguity, like a sign factor for example, local identifiability holds, and the FIM can be non-singular. Under weak conditions, local identifiability implies FIM regularity [8]:

Theorem 5 Assume that the FIM is of constant rank in the neighborhood of $\theta$. If $\theta$ is locally identifiable, then the FIM is regular at $\theta$.

And so we have the following theorem:

Theorem 6 Assume that the FIM is locally of constant rank at point $\theta$, then $\theta$ is locally identifiable if and only if the FIM is regular at $\theta$.

For the blind channel estimation problem, we shall see (sections 6.2.3 and 6.3) that this equivalence holds without the local rank assumption for the FIMs. 


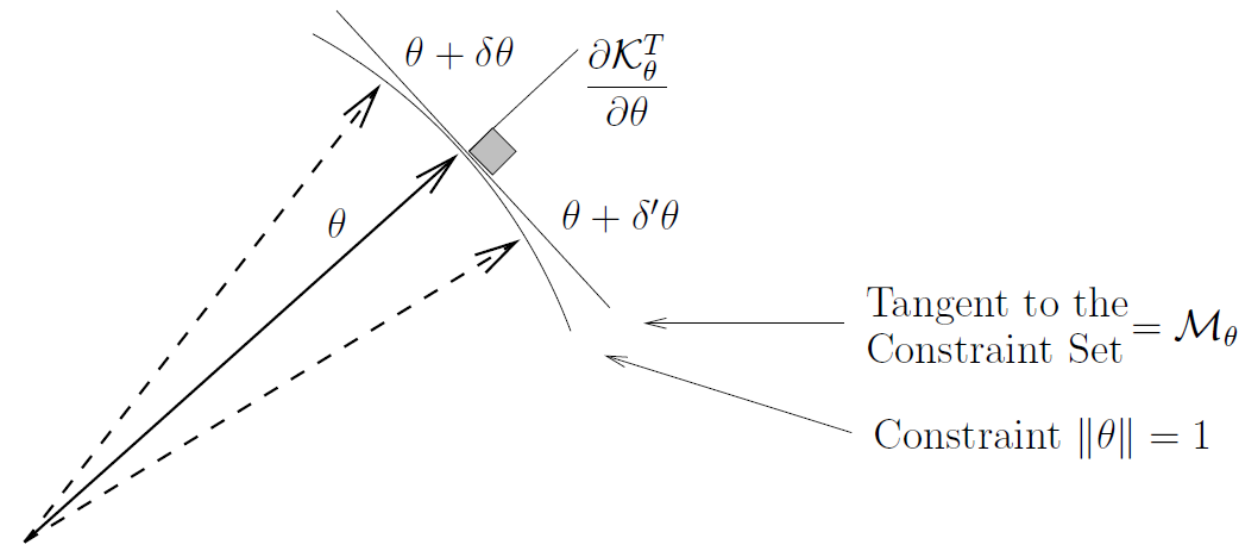

Figure 1: Example with constraint $|\theta|=1$.

\subsection{Blind Estimation}

Blind estimation (to be introduced further below) does not allow to identify the parameters. They are at best identifiable up to a certain blind ambiguity, a scale or a phase factor in our examples, which will be continuous in general, resulting in a singular FIM. Blind identifiability [1] is defined as in the regular case except that the condition is for the parameters to be identifiable up to the blind ambiguity.

In the deterministic and Gaussian input cases, local blind identifiability will be guaranteed if and only if the FIM has as many singularities as the number of continuous blind ambiguities, see Table 1. Furthermore, there will be as many independent constraints needed as the number of singularities to

\begin{tabular}{|c||c|c|}
\hline $\begin{array}{c}\text { Number of Real } \\
\text { Continuous Ambiguities }\end{array}$ & $\begin{array}{c}\text { Deterministic } \\
\text { Input }\end{array}$ & $\begin{array}{c}\text { Gaussian } \\
\text { Input }\end{array}$ \\
\hline \hline Real Data & 1 & 0 \\
\hline Complex Data & 2 & 1 \\
\hline
\end{tabular}

Table 1: Number of Continuous Ambiguities for the Deterministic and Gaussian Model

regularize the estimation problem.

\section{CRBs for Estimation with Constraints}

In this section, we consider real parameters (hence $\theta$ stands for $\theta_{R}$ if $\theta$ is complex). When the estimation is (locally) unidentifiable, the FIM is singular and the classical CRB result (2) cannot be applied; e.g. the channel cannot be estimated by blind estimation and the CRB is then in fact trivially $+\infty$.

In order to characterize the non regular estimation performance, we define regularized CRBs for which a certain a priori knowledge on the parameter $\theta$, under the form of a certain set of equality constraints, is assumed: this set of constraints should allow to adjust the parameters that cannot be identified and then to regularize the estimation problem. The introduction of a priori information on $\theta$ requires knowledge of $\theta$ in general, which is not available in practice. However, the point here is to evaluate the estimation performance (e.g. to compare different estimation algorithms), which implies that we compare $\hat{\theta}$ to the true $\theta$ which hence needs to be available. The sample estimation error covariance matrix will furthermore be compared to the CRB which also depends on $\theta$.

We determine a $\mathrm{CRB}$ for estimation under constraints for both cases of regular and singular unconstrained estimation problems. These results are also used in [3, to compare blind and semi-blind channel estimation performance under the same constraints.

CRBs for parametric estimation under constraints were derived in [4] in the case where the unconstrained estimation problem is regular. A simpler derivation of these results was presented in [10. The main ingredient of this simpler derivation was used in 11] to give an alternative expression for the CRB in the case where the unconstrained problem is unidentifiable. We shall succinctly restate these results, which appeared already in 12 for the case of linear constraints. In these references, and also here, we shall assume that the constraints are sufficient to regularize the estimation problem, i.e. to render the 
CRB finite. So, consider a $k$-fold constraint of the form:

$$
\mathcal{K}_{\theta}=0
$$

where $\mathcal{K}_{\theta}: \mathbb{R}^{n} \rightarrow \mathbb{R}^{k}$ is continuously differentiable and $k<n, n$ being the number of parameters in the vector $\theta$. A constrained parameter estimator $\hat{\theta}$ is called unbiased if it satisfies the constraints $\left(\mathcal{K}_{\hat{\theta}}=0\right)$ and if the parameter estimation bias is zero for all parameter values that satisfy the constraints 10 . The constrained CRB depends on the constraints only through the tangents to the constraint set at the true value of $\theta$ :

$$
\mathcal{M}_{\theta}=\left\{Z \in \mathbb{R}^{n} ; Z^{T} \frac{\partial \mathcal{K}_{\theta}^{T}}{\partial \theta}=0\right\} .
$$

We note that $\operatorname{dim}\left(\mathcal{M}_{\theta}\right)$ can be larger than $n-k$; the results of [10] can be generalized to the case in which the constraints are not independent. We can introduce a matrix $\mathcal{V}_{\theta}$ of full column rank such that $\mathcal{V}_{\theta}=\left(\frac{\partial \mathcal{K}_{\theta}^{T}}{\partial \theta}\right)^{\perp}$, meaning

$$
\text { range }\left\{\mathcal{V}_{\theta}\right\}=\mathcal{M}_{\theta}=\left(\operatorname{range}\left\{\frac{\partial \mathcal{K}_{\theta}^{T}}{\partial \theta}\right\}\right)^{\perp} .
$$

The key step now [10] is that the unbiasedness leads to a particular correlation between the parameter estimation error and the loglikelihood gradient restricted to $\mathcal{M}_{\theta}$ :

Lemma 1 Assume the estimator $\hat{\theta}$ and the true parameter $\theta$ satisfy the constraints, then unbiasedness implies

$$
E \mathcal{V}_{\theta}^{T} \frac{\partial \ln f(\boldsymbol{Y} \mid \theta)}{\partial \theta}(\hat{\theta}-\theta)^{T}=\mathcal{V}_{\theta}^{T}
$$

Using this lemma, the CRB derivation becomes an application of the following theorem.

Theorem 7 (Cauchy-Schwartz inequality for correlation matrices) Let $X_{1}$ and $X_{2}$ be random vectors with correlation matrices $R_{i j}=E X_{i} X_{j}^{T}, i, j=1,2$. Assume that $R_{11}$ is nonsingular. Then

$$
E\left(X_{2}-R_{21} R_{11}^{-1} X_{1}\right)\left(X_{2}-R_{21} R_{11}^{-1} X_{1}\right)^{T}=R_{22}-R_{21} R_{11}^{-1} R_{12} \geq 0
$$

with equality iff $X_{2}=R_{21} R_{11}^{-1} X_{1}$ in m.s.

With $X_{2}=\hat{\theta}-\theta$ and $X_{1}=\mathcal{V}_{\theta}^{T} \frac{\partial \ln f(\boldsymbol{Y} \mid \theta)}{\partial \theta}$, this leads immediately to the following main result.

Theorem 8 (Constrained CRB) Assume the constrained estimator $\hat{\theta}$ to be unbiased $(\hat{\theta}$ and $\theta$ satisfy the constraints $\mathcal{K}_{\theta}=0$ ), then

$$
C_{\tilde{\theta} \tilde{\theta}} \geq C R B_{C}=\mathcal{V}_{\theta}\left(\mathcal{V}_{\theta}^{T} \mathcal{J}_{\theta \theta} \mathcal{V}_{\theta}\right)^{-1} \mathcal{V}_{\theta}^{T}
$$

with equality iff

$$
\hat{\theta}-\theta=C R B_{C} \frac{\partial \ln f(\boldsymbol{Y} \mid \theta)}{\partial \theta} \text { in m.s. }
$$

A necessary and sufficient condition for the boundedness of $C R B_{C}$ is the nonsingularity of $\mathcal{V}_{\theta}^{T} \mathcal{J}_{\theta \theta} \mathcal{V}_{\theta}$.

\subsection{Interpretations and Remarks}

The key points to understand how constrained CRBs work are:

- the constrained CRB depends on the constraints only locally (as the CRB is based on derivatives),

- locally, the constraint set can be linearized.

To make things clearer, we distinguish between the variable $\theta$ and its true value $\theta^{\circ}$. Locally, a point $\theta$ belonging to the constraint set can be approximated as $\theta=\theta^{o}+\tilde{\theta}$, where $\tilde{\theta}$ belongs to $\mathcal{M}_{\theta}$, i.e. :

$$
\theta=\theta^{o}+\mathcal{V}_{\theta^{\circ}} \xi
$$

In figure 1. we show an example with constraint $|\theta|=1$ (for a complex $\theta$ with $n=1)$. 
From $(28)$ and applying the chain rule, we get

$$
\mathcal{J}_{\xi \xi}=\left(\frac{\partial \theta^{T}}{\partial \xi}\right) \mathcal{J}_{\theta \theta}\left(\frac{\partial \theta^{T}}{\partial \xi}\right)^{T}=\mathcal{V}_{\theta^{\circ}}^{T} \mathcal{J}_{\theta \theta} \mathcal{V}_{\theta^{\circ}}
$$

The estimation of $\xi$ is regular provided that $\mathcal{V}_{\theta^{\circ}}^{T} \mathcal{J}_{\theta \theta} \mathcal{V}_{\theta^{\circ}}$ is nonsingular. If we now get back to the initial parameter $\theta=\theta^{\circ}+\mathcal{V}_{\theta^{\circ}} \xi$, using the CRB for a transformation of parameters [7, we find:

$$
C R B_{\theta \theta}=\left(\frac{\partial \theta^{T}}{\partial \xi}\right)^{T} \mathcal{J}_{\xi \xi}^{-1}\left(\frac{\partial \theta^{T}}{\partial \xi}\right)=\mathcal{V}_{\theta^{\circ}}\left(\mathcal{V}_{\theta^{\circ}}^{T} \mathcal{J}_{\theta \theta} \mathcal{V}_{\theta^{\circ}}\right)^{-1} \mathcal{V}_{\theta^{\circ}}^{T}=C R B_{C}
$$

We see that the constrained CRB can be interpreted in terms of regular estimation: $\mathcal{V}_{\theta}^{T} \mathcal{J}_{\theta \theta} \mathcal{V}_{\theta}$ can be considered as the FIM for a minimal parameterization $\xi$ of $\theta$, and the results of equivalence mentioned in section 4 between FIM and local identifiability could also be applied here.

The $C R B_{C}$ is independent of the choice of $\mathcal{V}_{\theta}$ and can in fact also be written as:

$$
C R B_{C}=A_{\theta}\left(A_{\theta}^{T} \mathcal{J}_{\theta \theta} A_{\theta}\right)^{+} A_{\theta}^{T}
$$

where $A_{\theta}$ is a $n \times q$ matrix, $q \geq \operatorname{dim}\left(\mathcal{M}_{\theta}\right)$, such that $\mathcal{M}_{\theta}=\operatorname{range}\left\{A_{\theta}\right\}$. In particular, denoting $\frac{\partial \mathcal{K}_{\theta}^{T}}{\partial \theta}=\mathcal{K}_{\theta}^{\prime}$, we can take $A_{\theta}=P_{\mathcal{K}_{\theta}^{\prime}}^{\perp}=P_{\mathcal{V}_{\theta}}\left(\right.$ where $\left.P_{X}^{\perp}=I-P_{X}=I-X\left(X^{H} X\right)^{+} X^{H}\right)$ and obtain

$$
C R B_{C}=P_{\mathcal{V}_{\theta}}\left(P_{\mathcal{V}_{\theta}} \mathcal{J}_{\theta \theta} P_{\mathcal{V}_{\theta}}\right)^{+} P_{\mathcal{V}_{\theta}}=\left(P_{\mathcal{V}_{\theta}} \mathcal{J}_{\theta \theta} P_{\mathcal{V}_{\theta}}\right)^{+}
$$

There is a direct correspondence between the number of FIM singularities and the number of constraints necessary to have a finite constrained CRB, which is also the number of constraints necessary to have local identifiability.

Theorem 9 For the constrained CRB to be defined, it is necessary and sufficient to fulfill the following conditions.

- The number of independent constraints should be at least equal to $n-r\left(r=\operatorname{rank}\left(\mathcal{J}_{\theta \theta}\right)\right)$.

- At least $n-r$ independent columns of $\frac{\partial \mathcal{K}_{\theta}^{T}}{\partial \theta}$ should not be orthogonal to the null space of $\mathcal{J}_{\theta \theta}$.

A constraint of the form $\mathcal{K}_{\theta}=0$ has only a local effect and can be locally linearized

Theorem 10 The constrained $C R B$ (26) can also be interpreted as the CRB under the linear constraint:

$$
\left.\theta^{T} \frac{\partial \mathcal{K}_{\theta}^{T}}{\partial \theta}\right|_{\theta=\theta^{\circ}}=\left.\theta^{o T} \frac{\partial \mathcal{K}_{\theta}^{T}}{\partial \theta}\right|_{\theta=\theta^{\circ}}
$$

which means that the components of $\theta$ in $\operatorname{span}\left\{\left.\frac{\partial \mathcal{K}_{\theta}^{T}}{\partial \theta}\right|_{\theta=\theta^{\circ}}\right\}$ are known.

\subsection{Minimal constrained CRB}

\subsubsection{Constraint on the Global Parameter}

We assume here that $\mathcal{J}_{\theta \theta}$ is singular. When range $\left\{\mathcal{V}_{\theta}\right\}=\operatorname{range}\left\{\mathcal{J}_{\theta \theta}\right\}$ and since $\mathcal{V}_{\theta}$ has full column rank, $\mathcal{V}_{\theta}^{T} \mathcal{J}_{\theta \theta} \mathcal{V}_{\theta}$ is regular (minimal number of independent constraints in this case) and the constrained $\mathrm{CRB}$ is:

$$
C R B_{C}=\mathcal{J}_{\theta \theta}^{+} .
$$

This is a particular constrained CRB: we prove in Appendix A that, among all sets of a minimal number of independent constraints, $C R B_{C}=\mathcal{J}_{\theta \theta}^{+}$yields the lowest value for $\operatorname{tr}\left\{C R B_{C}\right\}$. This means that if we want to introduce a priori information in the form of independent constraints, enough to regularize the estimation problem, but not more (minimal number), then all the constraints should concentrate on the unidentifiable part of the parameters (range $\left\{\frac{\partial \mathcal{K}_{\theta}^{T}}{\partial \theta}\right\}=\operatorname{null}\left\{\mathcal{J}_{\theta \theta}\right\}$ ) to minimize $\operatorname{tr}\left\{C R B_{C}\right\}$. 


\subsubsection{Constraint on a Parameter Subset}

Consider the case of the joint estimation of two parameter vectors $\theta_{1}$ and $\theta_{2}$ of length $n_{1}$ and $n_{2}$ $\left(n_{1}+n_{2}=n\right)$. We are interested in the estimation of $\theta_{1}$ with $\theta_{2}$ being nuisance parameters. The overall parameter vector is $\theta=\left[\begin{array}{ll}\theta_{1}^{T} & \theta_{2}^{T}\end{array}\right]^{T}$.

$$
\mathcal{J}_{\theta \theta}=\left[\begin{array}{cc}
\mathcal{J}_{\theta_{1} \theta_{1}} & \mathcal{J}_{\theta_{1} \theta_{2}} \\
\mathcal{J}_{\theta_{2} \theta_{1}} & \mathcal{J}_{\theta_{2} \theta_{2}}
\end{array}\right]
$$

We consider the case in which $\mathcal{J}_{\theta \theta}$ is singular but $\mathcal{J}_{\theta_{2} \theta_{2}}$ is regular. To regularize the estimation problem, we consider the introduction of (independent) constraints on $\theta_{1}$ only: $\mathcal{K}_{\theta_{1}}=0, \mathcal{K}_{\theta_{1}}: \mathbb{R}^{n_{1}} \rightarrow \mathbb{R}^{k_{1}}$.

$$
\frac{\partial \mathcal{K}_{\theta_{1}}^{T}}{\partial \theta}=\left[\begin{array}{c}
\frac{\partial \mathcal{K}_{\theta_{1}}^{T}}{\partial \theta_{1}} \\
0_{n_{2}, k_{1}}
\end{array}\right]
$$

(assumed full column rank). Let $\mathcal{V}_{\theta_{1}}=\left(\frac{\partial \mathcal{K}_{\theta_{1}}}{\partial \theta_{1}}\right)^{\perp}$ be a $n_{1} \times\left(n_{1}-k_{1}\right)$ matrix of full column rank. We can choose

$$
\mathcal{V}_{\theta}=\left[\begin{array}{cc}
\mathcal{V}_{\theta_{1}} & 0_{n_{1}, n_{2}} \\
0_{n_{2}, n_{1}} & I_{n_{2}, n_{2}}
\end{array}\right] \text {. }
$$

The constrained CRB for $\theta$ is:

$$
C R B_{C}=\mathcal{V}_{\theta}\left(\mathcal{V}_{\theta}^{H} \mathcal{J}_{\theta \theta} \mathcal{V}_{\theta}\right)^{-1} \mathcal{V}_{\theta}^{H}=\mathcal{V}_{\theta}\left[\begin{array}{cc}
\mathcal{V}_{\theta_{1}}^{H} \mathcal{J}_{\theta_{1} \theta_{1}} \mathcal{V}_{\theta_{1}} & \mathcal{V}_{\theta_{1}}^{H} \mathcal{J}_{\theta_{1} \theta_{2}} \\
\mathcal{J}_{\theta_{2} \theta_{1}} \mathcal{V}_{\theta_{1}} & \mathcal{J}_{\theta_{2} \theta_{2}}
\end{array}\right]^{-1} \mathcal{V}_{\theta}^{H}
$$

and the constrained CRB for $\theta_{1}$ separately is:

$$
C R B_{C, \theta_{1}}=\mathcal{V}_{\theta_{1}}\left(\mathcal{V}_{\theta_{1}}^{H}\left[\mathcal{J}_{\theta_{1} \theta_{1}}-\mathcal{J}_{\theta_{1} \theta_{2}} \mathcal{J}_{\theta_{2} \theta_{2}}^{-1} \mathcal{J}_{\theta_{2} \theta_{1}}\right] \mathcal{V}_{\theta_{1}}\right)^{-1} \mathcal{V}_{\theta_{1}}^{H}=\mathcal{V}_{\theta_{1}}\left(\mathcal{V}_{\theta_{1}}^{H} \mathcal{J}_{\theta_{1} \theta_{1}}(\theta) \mathcal{V}_{\theta_{1}}\right)^{-1} \mathcal{V}_{\theta_{1}}^{H}
$$

where we introduced the notation $\mathcal{J}_{\theta_{1} \theta_{1}}(\theta)$ for $\mathcal{J}_{\theta_{1} \theta_{1}}-\mathcal{J}_{\theta_{1} \theta_{2}} \mathcal{J}_{\theta_{2} \theta_{2}}^{-1} \mathcal{J}_{\theta_{2} \theta_{1}}$. This notation will be reused below. Note that $\mathcal{J}_{\theta_{1} \theta_{1}}^{-1}(\theta)$ would be the unconstrained CRB for $\theta_{1}$ if $\mathcal{J}_{\theta \theta}$ were regular. Note that with $\mathcal{J}_{\theta_{2} \theta_{2}}$ being regular, $\mathcal{J}_{\theta_{1} \theta_{1}}(\theta)$ has the same number of singularities as $\mathcal{J}_{\theta \theta}$ in the singular case. Now assume that the constraints are such that range $\left\{\mathcal{V}_{\theta_{1}}\right\}=$ range $\left\{\mathcal{J}_{\theta_{1} \theta_{1}}(\theta)\right\}$. Then it can be proven that such constraints give the minimal constrained CRB for $\theta_{1}$,

$$
C R B_{C, \theta_{1}}=\mathcal{J}_{\theta_{1} \theta_{1}}^{+}(\theta)
$$

over all sets of a minimal number of independent constraints on $\theta_{1}$ only.

\section{CRBs for Blind FIR Multichannel Estimation}

These results are now applied to blind FIR multichannel estimation. Two models are presented here: the deterministic model which considers the symbols as deterministic quantities and the Gaussian model which considers them as Gaussian random variables [1. We first present the multichannel model, which is fundamental in blind channel estimation (from second-order statistics).

\subsection{The Multichannel Model}

We consider a single-user multichannel model: see [1] for more details on this model. Let $a(k)$ be a sequence of symbols received through $m$ channels of length $\mathrm{N}$ and with impulse response $\boldsymbol{h}(i)$ :

$$
\boldsymbol{y}(k)=\sum_{i=0}^{N-1} \boldsymbol{h}(i) a(k-i)+\boldsymbol{v}(k),
$$

$\boldsymbol{y}(k)=\left[y_{1}(k) \cdots y_{m}(k)\right]^{T}$, and similarly for $\boldsymbol{h}(k)$ and $\boldsymbol{v}(k) . \boldsymbol{v}(k)$ is an additive independent white Gaussian noise with $r \boldsymbol{v} \boldsymbol{v}(k-i)=\mathrm{E} \boldsymbol{v}(k) \boldsymbol{v}(i)^{H}=\sigma_{v}^{2} I_{m} \delta_{k i}$ and when $\boldsymbol{v}(k)$ is complex $\mathrm{E} \boldsymbol{v}(k) \boldsymbol{v}^{T}(i)=0$ (circular noise). 
Throughout the paper, we will distinguish between complex and real symbols. For real symbols, it will be advantageous to consider separately the real and imaginary parts of the channel and received signal:

$$
\left[\begin{array}{l}
\operatorname{Re}\left(y_{l}(k)\right) \\
\operatorname{Im}\left(y_{l}(k)\right)
\end{array}\right]=\sum_{i=0}^{N-1}\left[\begin{array}{l}
\operatorname{Re}\left(h_{l}(i)\right) \\
\operatorname{Im}\left(h_{l}(i)\right)
\end{array}\right] a(k-i)+\left[\begin{array}{l}
\operatorname{Re}\left(v_{l}(k)\right) \\
\operatorname{Im}\left(v_{l}(k)\right)
\end{array}\right], l=1, \ldots, n
$$

where $n$ now denotes the product of the oversampling factor and the number of sensors. The vector signals now become $\boldsymbol{y}(k)=\left[\operatorname{Re}\left(y_{1}(k)\right) \operatorname{Im}\left(y_{1}(k)\right) \cdots \operatorname{Re}\left(y_{n}(k)\right) \operatorname{Im}\left(y_{n}(k)\right)\right]^{T}$ and similarly for $\boldsymbol{h}(k)$ and $\boldsymbol{v}(k)$. This leads to a representation similar to (41). The number of channels gets doubled, which has for advantage to increase diversity. Note that the monochannel case does not exist in the real case, unless transmission is performed in baseband.

Assume we receive $M$ samples, concatenated in the vector $\boldsymbol{Y}_{M}(k)$ :

$$
\boldsymbol{Y}_{M}(k)=\mathcal{T}_{M}(h) A_{M+N-1}(k)+\boldsymbol{V}_{M}(k)
$$

where $\boldsymbol{Y}_{M}(k)=\left[\boldsymbol{y}^{T}(k) \cdots \boldsymbol{y}^{T}(k-M+1)\right]^{T}$, and $\boldsymbol{V}_{M}(k)$ and $A_{M}(k)$ are similarly defined. $\mathcal{T}_{M}(h)$ is a block Toeplitz matrix with $M$ block rows and $\left[\begin{array}{ll}\boldsymbol{H} & 0_{m \times(M-1)}\end{array}\right]$ as first block row:

$$
\boldsymbol{H}=[\boldsymbol{h}(0) \cdots \boldsymbol{h}(N-1)] \text { and } h=\left[\boldsymbol{h}^{T}(0) \cdots \boldsymbol{h}^{T}(N-1)\right]^{T} .
$$

We furthermore denote $\mathbf{H}(z)=\sum_{i=0}^{N-1} \boldsymbol{h}(i) z^{-i}=\left[\mathrm{H}_{1}(z) \cdots \mathrm{H}_{m}(z)\right]^{T}$, the SIMO channel transfer function. We shall simplify the notation in 43 with $k=M-1$ to:

$$
\boldsymbol{Y}=\mathcal{T}(h) A+\boldsymbol{V} .
$$

Commutativity of Convolution We will need the convolution commutativity relationship:

$$
\mathcal{T}(h) A=\mathcal{A} h
$$

where: $\mathcal{A}=A^{\prime} \otimes I_{m}$,

$$
A^{\prime}=\left[\begin{array}{cccc}
a(-N+1) & a(-N+2) & \cdots & a(0) \\
a(-N+2) & \cdot & . & \vdots \\
\vdots & . & . & \vdots \\
a(M-N+2) & \cdots & \cdots & a(M-1)
\end{array}\right]
$$

Irreducible and Reducible Channels A channel is said irreducible if its subchannels $\mathrm{H}_{i}(z)$ have no zeros in common, and reducible otherwise. A reducible channel can be decomposed as:

$$
\mathbf{H}(z)=\mathbf{H}_{I}(z) \mathrm{H}_{c}(z),
$$

where $\mathbf{H}_{I}(z)$ of length $N_{I}$ is irreducible and $\mathrm{H}_{c}(z)$ of length $N_{c}=N-N_{I}+1$ is a monochannel.

\subsection{Deterministic Model}

The deterministic model considers the joint estimation of the unknown input symbols $A$ and the channel coefficients $h$. The parameter vector is $\theta=\left[A^{T} h^{T}\right]^{T}$. It can indeed be shown that the estimation of $A$ and $h$ is decoupled from that of $\sigma_{v}^{2}$, at the FIM level (and also from an estimation point of view). So we shall omit $\sigma_{v}^{2}$ in $\theta$. Identifiability of $(A, h)$ occurs from $m_{Y}(\theta)=\boldsymbol{X}=\mathcal{T}(h) A$, the signal part of $\boldsymbol{Y}$, whereas identifiability of $\sigma_{v}^{2}$ occurs from $C_{Y Y}(\theta)=\sigma_{v}^{2} I$. Blind identifiability in the deterministic model requires the channel to be irreducible and the burst length and the number of input excitation modes to satisfy certain minimum requirements [1].

\subsubsection{FIMs}

Circular Complex Input Constellation As $\boldsymbol{Y}$ is circular, we can work with the complex probability density function of the Gaussian random variable $\boldsymbol{Y} \sim \mathcal{N}\left(\mathcal{T}(h) A, \sigma_{v}^{2} I\right)$. 
As $J_{\theta \theta^{*}}=0$, the complex FIM $J_{\theta \theta}$ is equivalent to the real one $\mathcal{J}_{\theta_{R} \theta_{R}}$ and is equal to:

$$
J_{\theta \theta}=\frac{1}{\sigma_{v}^{2}}\left(\frac{\partial \boldsymbol{X}^{H}}{\partial \theta^{*}}\right)\left(\frac{\partial \boldsymbol{X}^{H}}{\partial \theta^{*}}\right)^{H}=\frac{1}{\sigma_{v}^{2}}\left[\begin{array}{c}
\mathcal{T}^{H}(h) \\
\mathcal{A}^{H}
\end{array}\right]\left[\begin{array}{cc}
\mathcal{T}(h) & \mathcal{A}
\end{array}\right]
$$

because $\frac{\partial \boldsymbol{X}^{H}}{\partial A^{*}}=\mathcal{T}^{H}(h)$ and $\frac{\partial \boldsymbol{X}^{H}}{\partial h^{*}}=\mathcal{A}^{H}$.

Real Symbol Constellation The FIM is the same as in 490 . This equality of the expressions will allow us to treat the complex and real cases simultaneously.

\subsubsection{Singularities of the FIMs}

Under the blind deterministic identifiability conditions, $(h, A)$ are identifiable up to a scale factor: indeed $\mathcal{T}(h) A=\mathcal{T}(h / \alpha) \alpha A$. This results in one (complex) singularity of the complex FIM (see theorem below). We examine here the singularities in that case. The singularities of the FIM can be considered at the level of $\theta$ (joint estimation of $A$ and $h$ ) or at the level of $h$ (estimation of $h$ with $A$ considered as nuisance parameter).

Singularities of $J_{\theta \theta} . \quad J_{\theta \theta}=\frac{1}{\sigma_{v}^{2}}[\mathcal{T}(h) \mathcal{A}]^{H}\left[\begin{array}{ll}\mathcal{T}(h) & \mathcal{A}\end{array}\right]$ admits as null vector: $\theta_{s}=\left[\begin{array}{ll}-A^{T} & h^{T}\end{array}\right]^{T}$. Indeed, $[\mathcal{T}(h) \mathcal{A}]\left[\begin{array}{ll}-A^{T} & h^{T}\end{array}\right]^{T}=-\mathcal{T}(h) A+\mathcal{A} h=0$, by exploiting 466 . When $\mathcal{T}(h)$ and $\mathcal{A}$ have full column rank, the nullity of $J_{\theta \theta}$ is the dimension of the intersection of the column spaces of $\mathcal{T}(h)$ and $\mathcal{A}$, which is one.

Singularities of $J_{h h}(\theta) \triangleq \frac{1}{\sigma_{v}^{2}} \mathcal{A}^{H} P_{\mathcal{T}(h)}^{\perp} \mathcal{A}$. If $J_{h h}(\theta)$ were regular, its inverse would be the CRB for $h$. However, $J_{h h}(\theta)$ is singular. Indeed, assume that $h^{\prime}$ is a singular vector of $J_{h h}(\theta): \mathcal{A}^{H} P_{\mathcal{T}(h)}^{\perp} \mathcal{A} h^{\prime}=0$. Then, as $\mathcal{A}$ has full column rank, this means that $\mathcal{A} h^{\prime} \in \operatorname{range}\{\mathcal{T}(h)\}$ : there exists an $A^{\prime}$ such that $\mathcal{A} h^{\prime}=\mathcal{T}\left(h^{\prime}\right) A=\mathcal{T}(h) A^{\prime}$. This last relation is satisfied for $\left(h^{\prime}, A^{\prime}\right)=(h, A)$. Hence, $J_{h h}(\theta)$ has one singularity with $h$ as its singular vector $\left(\mathcal{A}^{H} P_{\mathcal{T}(h)}^{\perp} \mathcal{A} h=\mathcal{A}^{H} P_{\mathcal{T}(h)}^{\perp} \mathcal{T}(h) A=0\right)$ and the singularity of $J_{h h}(\theta)$ is due to the same mechanism that leads to the singularity of the global FIM $J_{\theta \theta}$.

In the complex case, $\mathcal{J}_{\theta_{R} \theta_{R}}$ will have 2 singularities spanned by:

$$
h_{S_{1}}=\left[\begin{array}{c}
\operatorname{Re}(h) \\
\operatorname{Im}(h)
\end{array}\right]=h_{R} \quad \text { and } \quad h_{S_{2}}=\left[\begin{array}{r}
-\operatorname{Im}(h) \\
\operatorname{Re}(h)
\end{array}\right]=\left[\begin{array}{l}
\operatorname{Re}(j h) \\
\operatorname{Im}(j h)
\end{array}\right] .
$$

The first null vector can be interpreted as corresponding to the ambiguity in the norm of the channel and the second one to the ambiguity in the phase factor.

\subsubsection{Equivalence between FIM Regularity and Local Identifiability}

The link between blind identifiability and FIM singularities in the specific case of the blind deterministic model was already studied in [13, 14]:

Theorem 11 For $M \geq 2(N-1)$, or $M \geq N$ for 2 subchannels $(m=2)$, a channel is blindly identifiable up to a scale factor if and only if the complex FIM $J_{\theta \theta}$ has exactly one singularity.

Proof: see [14.

In general, there is a correspondence between local identifiability and FIM regularity.

Theorem 12 A channel is locally blindly identifiable up to a scale factor if and only if the complex FIM $J_{\theta \theta}$ has exactly one singularity. 
Proof: Assume that the FIM has a singular vector $\theta^{\prime}=\left[\begin{array}{ll}h^{\prime T} & A^{\prime T}\end{array}\right]^{T}$ different from $\left[\begin{array}{ll}h^{T} & -A^{T}\end{array}\right]^{T}$ :

$$
\mathcal{T}(h) A^{\prime}+\mathcal{T}\left(h^{\prime}\right) A=0 \text {. }
$$

Then for $\epsilon>0$ arbitrarily small:

$$
\begin{aligned}
m_{Y}\left(\theta+\epsilon \theta^{\prime}\right)-m_{Y}(\theta) & =\mathcal{T}\left(h+\epsilon h^{\prime}\right)\left[A+\epsilon A^{\prime}\right]-\mathcal{T}(h) A \\
& =\epsilon\left[\mathcal{T}(h) A^{\prime}+\mathcal{T}\left(h^{\prime}\right) A\right]+O\left(\epsilon^{2}\right) \\
& =O\left(\epsilon^{2}\right)
\end{aligned}
$$

which implies that $\theta$ is not locally blindly identifiable.

Now assume that $\theta$ is not locally blindly identifiable, then one can find $\Delta h$ and $\Delta A$, where $\|\Delta h\|$ and $\|\Delta A\|$ are arbitrarily small, and not simultaneously colinear with $h$ and $A$ resp. verifying $\mathcal{T}(h) A=$ $\mathcal{T}(h+\Delta h)(A+\Delta A)$. Then:

$$
\begin{aligned}
\mathcal{T}(h+\Delta h)(A+\Delta h)-\mathcal{T}(h) A & =\mathcal{A} \Delta h+\mathcal{T}(h) \Delta A+O(\|\Delta h\|\|\Delta A\|) \\
& =0 .
\end{aligned}
$$

This implies that $\left[\begin{array}{lll}\Delta h^{T} & \Delta A^{T}\end{array}\right]^{T}$ is a singular vector of the FIM, non colinear to $\left[\begin{array}{ll}h^{T} & -A^{T}\end{array}\right]^{T}$.

Using a similar derivation, we can also show the equivalence between the regularity of $\mathcal{V}_{\theta}^{H} J_{\theta \theta} \mathcal{V}_{\theta}$ and local identifiability under constraint $\mathcal{K}_{\theta}$ :

Theorem 13 The estimation problem under constraint $\mathcal{K}_{\theta}$ is locally identifiable if and only if the regularized FIM $\mathcal{V}_{\theta}^{H} J_{\theta \theta} \mathcal{V}_{\theta}$ (with definitions of section 5 ) is regular.

The same theorem will hold for the Gaussian model in section 6.3 also but will not be restated there.

\subsubsection{Regularized Blind CRB}

To define a regularized blind CRB, we assume some a priori knowledge. Different forms of a priori knowledge are possible. A technique often used consists in assuming a coefficient of the channel to be known. This is however not robust as performance depends heavily on the choice of this known coefficient (which can be arbitrarily small). The proposed regularized CRB, the Moore-Penrose pseudo-inverse of the FIM, appears less arbitrary and is directly related to the blind scale factor ambiguity.

Blind methods commonly consider the quadratic constraint: $h^{H} h=1$ (see [1]). This constraint does not render the problem identifiable: it leaves a sign ambiguity when $h$ is real and a continuous phase ambiguity when $h$ is complex. In the former case, the computation of mean squared error (MSE) assumes the right sign (the right sign could be taken as the sign giving the smallest error). In the complex case however, which phase factor should be chosen? A frequent choice consists in imposing one element of $h$ to be real and positive; again the resulting CRB depends on the choice of this element.

The blind regularized CRB proposed here is computed under the following constraints:

(1) A quadratic constraint:

$$
h^{H} h=h^{o H} h^{o}
$$

(equivalent to the usual constraint $h^{H} h=1$ ) which allows to adjust the norm of the channel.

(2) In the complex case, an additional constraint is necessary to adjust the phase factor:

$$
h_{S_{2}}^{o T} h_{R}=h_{S_{2}}^{o T} h_{R}^{o}=0 .
$$

In both real and complex cases, these constraints leave a sign ambiguity which does not lead to FIM singularity. For MSE evaluation, the ambiguity can be resolved by requiring $h^{o T} h>0$.

Result 1 Under constraint (54) (and (55) for the complex case) the CRB for $h$ is the Moore-Penrose pseudo-inverse of $J_{h h}(\theta)$ :

$$
C R B_{C, h}=J_{h h}^{+}(\theta)=\sigma_{v}^{2}\left(\mathcal{A}^{H} P_{\mathcal{T}(h)}^{\perp} \mathcal{A}\right)^{+}
$$



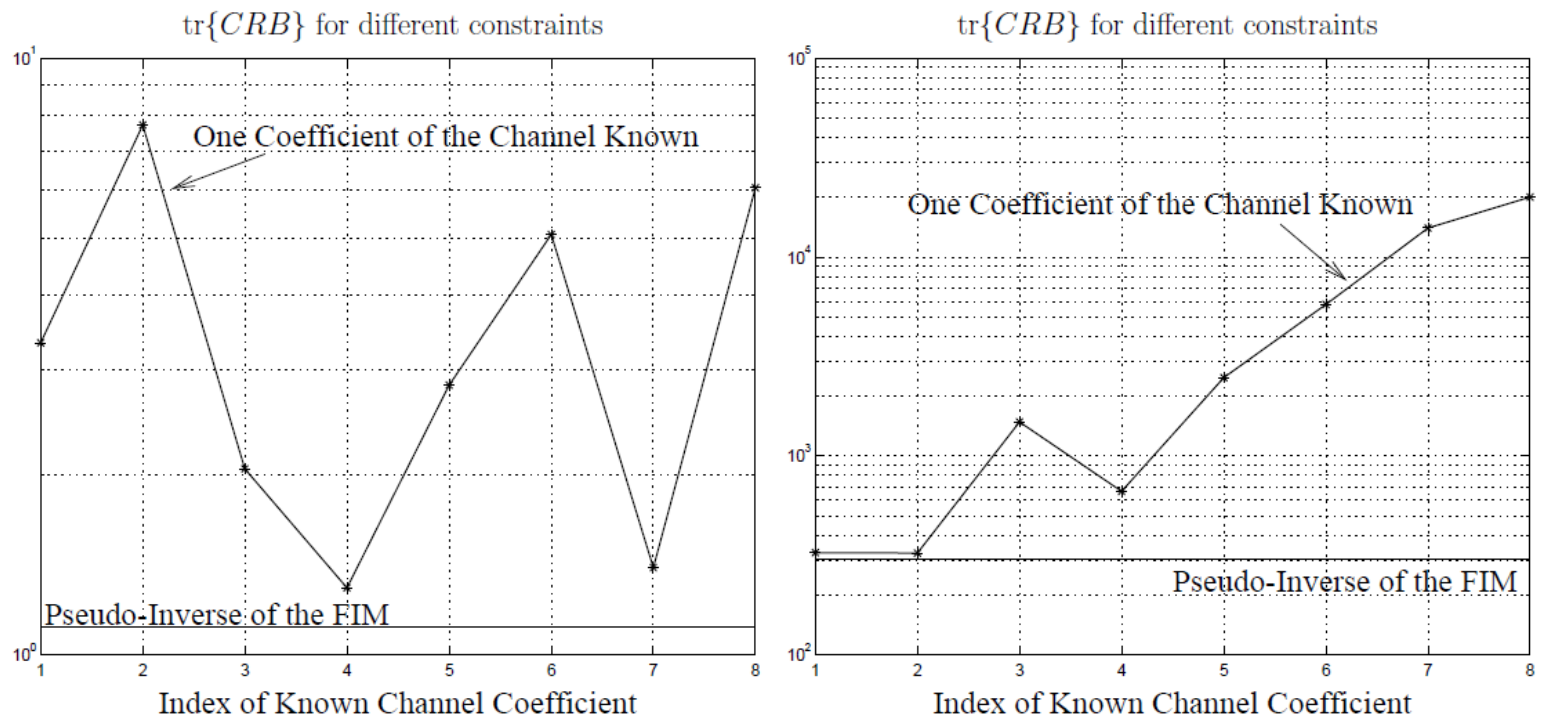

Figure 2: Comparison between CRBs with different a priori knowledge. The coefficients designate the coefficients of the vector $h$ for the random channel $\boldsymbol{H}_{1}$ (left) and the decreasing channel impulse response $\boldsymbol{H}_{2}$ (right).

Proof: Following the notations of section 5 the constraint is:

$$
\mathcal{K}_{h_{R}}=\left[\begin{array}{c}
h_{R}^{T} h_{R}-h_{R}^{o T} h_{R}^{o} \\
h_{S_{2}}^{o T} h_{R}
\end{array}\right]=0
$$

leading to

$$
\frac{\partial \mathcal{K}_{h_{R}}^{T}}{\partial h_{R}}=\left[\begin{array}{ll}
2 h_{R}^{o} & h_{S_{2}}^{o}
\end{array}\right] .
$$

As $h_{R}$ and $h_{s_{2}}$ are the singular vectors of $\mathcal{J}_{h_{R} h_{R}}(\theta)$ (which corresponds to the previously defined complex $\left.J_{h h}(\theta)\right)$, the orthogonal complement of range $\left\{\frac{\partial \mathcal{K}_{\theta_{R}}^{T}}{\partial h_{R}}\right\}$ equals range $\left\{\mathcal{J}_{h_{R} h_{R}}(\theta)\right\}$. According to section 5.2.2, the CRB under constraint 57) is:

$$
C R B_{C, h_{R}}=\mathcal{J}_{h_{R} h_{R}}^{+}(\theta)
$$

and the corresponding complex contrained CRB can be shown to be:

$$
C R B_{C, h}=J_{h h}^{+}(\theta)
$$

The choice of the first constraint is not only motivated by its common use. As mentioned in section 5.2, this constraint (possibly combined with the linear constraint on the phase) leads to the minimal constrained CRB.

In Figure 2 we illustrate the importance of the choice for a constraint by comparing the proposed $\mathrm{CRB} \operatorname{tr}\left\{J_{h h}^{+}(\theta)\right\}$ to a constrained CRB for which one of the channel coefficients (of varying position) is assumed known. Two channels are tested: a randomly chosen channel and a channel with slowly decreasing coefficients:

$$
\begin{aligned}
\boldsymbol{H}_{1} & =\left[\begin{array}{rrrr}
0.9477 & -1.1156 & 1.1748 & 1.6455 \\
-0.5257 & -1.5923 & 0.4851 & -0.4542
\end{array}\right] \\
\boldsymbol{H}_{2} & =\left[\begin{array}{rrrr}
1.0000 & 0.5000 & -0.1500 & 0.0695 \\
1.5000 & -0.9500 & 0.3050 & 0.0550
\end{array}\right]
\end{aligned}
$$

One observes that when the channel coefficient which is assumed known is small, the corresponding $C R B_{C}$ can get quite large (arbitrarily large as the coefficient magnitude shrinks). 


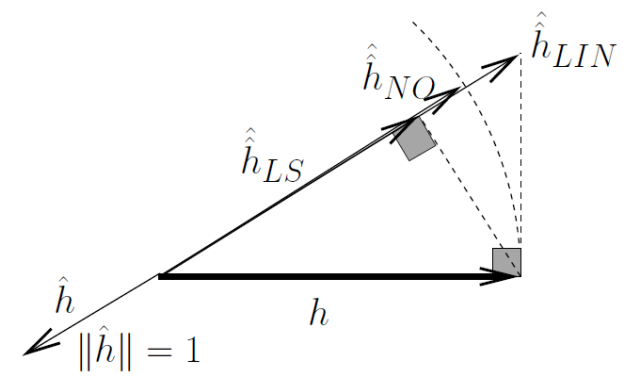

Figure 3: Deterministic case: asymptotically equivalent constraints.

Some Equivalent Constraints Another choice for the constraint, which leads to the same range $\left\{\frac{\partial \mathcal{K}_{h_{R}}^{T}}{\partial h_{R}}\right\}$ is the following linear constraint:

$$
h^{o H} h=h^{o H} h^{o}
$$

This constraint, which leaves no sign ambiguity, corresponds to forcing the components of $h$ in the nullspace of $J_{h h}$ to their true values.

Often, $h$ is estimated under a unit norm constraint $\|\hat{h}\|=1$, and the scale factor is adjusted in different ways. The following adjustments lead to the same minimal CRB.

- The norm of the channel is adjusted so that $\|\hat{h}\|=\left\|h^{o}\right\|$ and the phase using the phase constraint 55. We denote the resulting estimate $\hat{\hat{h}}_{N O}$.

- The scale factor is adjusted in the least-square sense [15, [16]: $\min _{\alpha}\left\|h^{o}-\alpha \hat{h}\right\|^{2}$. To be more precise, in this case the trace of the corresponding constrained $\mathrm{CRB}$ is $\operatorname{tr}\left\{C R B_{C}\right\}$ of equation 56 .

Proof: The solution of the least-squares problem is $\hat{\hat{h}}_{L S}=\hat{\alpha} \hat{h}=P_{\hat{h}} h^{o}$. Then, $\Delta \hat{\hat{h}}=P_{\hat{h}} h^{o}-h^{o}=$ $-P_{\hat{h}}^{\perp} h^{o} ; C_{\Delta \hat{\hat{h}} \Delta \hat{\hat{h}}}=\mathrm{E} P_{\hat{h}}^{\perp} h^{o} h^{o H} P_{\hat{h}}^{\perp}$.

$$
\begin{aligned}
& \operatorname{tr}\left\{C_{\Delta \hat{\hat{h}} \Delta \hat{\hat{h}}}\right\}=\operatorname{tr}\left\{\mathrm{E} P_{\hat{h}}^{\perp} P_{h^{o}}\right\}\left\|h^{o}\right\|^{2}=\operatorname{tr}\left\{\mathrm{E} P_{h^{\circ}}^{\perp} \hat{h} \hat{h}^{H}\right\}\left\|h^{o}\right\|^{2} \\
& =\operatorname{tr}\left\{\mathrm{E} P_{h^{\circ}}^{\perp}\left(\hat{h}\left\|h^{o}\right\|\right)\left(\hat{h}\left\|h^{o}\right\|\right)^{H} P_{h^{\circ}}^{\perp}\right\} \\
& =\operatorname{tr}\left\{\mathrm{E} P_{h^{o}}^{\perp}\left(\Delta \hat{\hat{h}}_{N O}\right)\left(\Delta \hat{\hat{h}}_{N O}\right)^{H} P_{h^{o}}^{\perp}\right\}=\operatorname{tr}\left\{P_{h^{\circ}}^{\perp} C_{\Delta \hat{\hat{h}}_{N O} \Delta \hat{\hat{h}}_{N O}} P_{h^{\circ}}^{\perp}\right\} \\
& \geq \operatorname{tr}\left\{P_{h^{\circ}}^{\perp} C R B_{C, h} P_{h^{\circ}}^{\perp}\right\}=\operatorname{tr}\left\{C R B_{C, h}\right\}
\end{aligned}
$$

Another way to adjust the scale factor consists of adjusting $\alpha$ by the following linear constraint $h^{o H} \hat{\hat{h}}_{L I N}=h^{o H} \alpha \hat{h}=h^{o H} h^{o}$, leading to the following channel estimate:

$$
\hat{\hat{h}}_{L I N}=\frac{\hat{h} h^{o H}}{h^{o H} \hat{h}} h^{o} .
$$

When the estimation of $h$ is consistent, then, asymptotically, the CRB for this constrained channel estimate is the same $C R B_{C, h}$ of $(56)$.

In figure 3 , we show the solutions $\hat{\hat{h}}_{N O}, \hat{\hat{h}}_{L S}, \hat{\hat{h}}_{L I N}$ for a real channel of length $N=1$ and with 2 subchannels.

\subsubsection{Reducible Channel Case}

In this case, $\boldsymbol{H}(z)=\boldsymbol{H}_{I}(z) \mathrm{H}_{c}(z)$ where $\mathrm{H}_{c}(z)$ is a monic (first coefficient equal to 1) polynomial in $z^{-1}$. In the time domain, this becomes $h=T_{c} h_{I}$ where $T_{c}=\mathcal{T}_{N_{I}}^{T}\left(h_{c}\right) \otimes I_{m}$. This decomposition leads us to introduce $A_{I}=\mathcal{T}\left(h_{c}\right) A$, the input signal filtered by $\mathrm{H}_{c}(z)$ and we can write the noise-free received signal in the following ways

$$
\begin{aligned}
\boldsymbol{X}=\mathcal{T}(h) A & =\mathcal{T}\left(h_{I}\right) \mathcal{T}\left(h_{c}\right) A=\mathcal{T}\left(h_{I}\right) A_{I}=\mathcal{A}_{I} h_{I} \\
& =\mathcal{A} h=\mathcal{A} T_{c} h_{I}=\mathcal{A}_{I} h_{I}
\end{aligned}
$$


where $\mathcal{A}_{I}=\mathcal{A} T_{c}$. Since $\mathcal{T}(h)=\mathcal{T}\left(h_{I}\right) \mathcal{T}\left(h_{c}\right)$, we have $P_{\mathcal{T}(h)}=P_{\mathcal{T}\left(h_{I}\right)}$. In the reducible case, the quantities that are blindly identifiable are $h_{I}, A_{I}$, up to one scalar indeterminacy (assuming certain identifiability conditions for the burst length $M$ and the excitation modes in $A$ [1]).

To get $h=T_{c} h_{I}$ from $h_{I}$, there are $N_{c}-1$ indeterminacies (the coefficients of $h_{c}$ ). To get $A$ from $A_{I}=\mathcal{T}\left(h_{c}\right) A$, there are also $N_{c}-1$ indeterminacies (modes of $A$ that are potentially coinciding with zeros of $\mathrm{H}_{c}(z)$; alternatively, one needs $N_{c}-1$ "initial conditions" to get $A$ from $A_{I}$, given $h_{c}$ (which was already needed to get $h$ from $\left.h_{I}\right)$ ). So there are $2 N_{c}-1$ indeterminacies all in all and hence $J_{\theta \theta}$ has $2 N_{c}-1$ singularities. Now,

$$
J_{h h}(\theta)=\frac{1}{\sigma_{v}^{2}} \mathcal{A}^{H} P_{\mathcal{T}(h)}^{\perp} \mathcal{A}=\frac{1}{\sigma_{v}^{2}} \mathcal{A}^{H} P_{\mathcal{T}\left(h_{I}\right)}^{\perp} \mathcal{A}
$$

has $N_{c}$ singularities. Indeed, an alternative decomposition for $h$ is $h=T_{I} h_{c}$ where $T_{I}$ is block Toeplitz with $\left[\begin{array}{ll}h_{I}^{T} & 0_{1 \times\left(N_{c}-1\right) m}\end{array}\right]^{T}$ as first column. Now consider $h^{\prime}=T_{I} h_{c}^{\prime}$ where $h_{c}^{\prime}$ is arbitrary (not monic). Then $\mathcal{A} h^{\prime}=\mathcal{T}\left(h^{\prime}\right) A=\mathcal{T}\left(h_{I}\right) \mathcal{T}\left(h_{c}^{\prime}\right) A$. Hence $J_{h h}(\theta) h^{\prime}=0$ and null $\left\{J_{h h}(\theta)\right\}=$ range $\left\{T_{I}\right\}$. So we get:

Result 2 The CRB for estimating a reducible $h$ under the constraint $T_{I}^{o H} h=T_{I}^{o H} h^{o}$ is

$$
C R B_{C, h}=J_{h h}^{+}(\theta)=\sigma_{v}^{2}\left(\mathcal{A}^{H} P_{\mathcal{T}(h)}^{\perp} \mathcal{A}\right)^{+} .
$$

Note that this set of constraints implies in particular $h^{o H} h=h^{o H} h^{o}$. Note also that under these constraints, an estimate $\widehat{h}$ will not necessarily be of the form $\widehat{h}=\widehat{T}_{I} \widehat{h}_{c}$ with $\widehat{h}_{c}$ equal to $h_{c}$ or not: $\widehat{h}$ is not necessarily reducible. Nevertheless, the constraints mentioned are sufficient to make $h$ identifiable. Indeed, identifiability of $h$ with deterministic symbols implies being able to determine $h$ from the noisefree signal. If we do that with for instance the subspace fitting method, then the signal subspace will be range $\left\{\mathcal{T}\left(h_{I}^{o}\right)\right\}$. Subspace fitting will force range $\{\mathcal{T}(\widehat{h})\} \subset$ range $\left\{\mathcal{T}\left(h_{I}^{o}\right)\right\}$ which implies $\widehat{\boldsymbol{H}}(z)=$ $\boldsymbol{H}_{I}^{o}(z) \widehat{\mathrm{H}}_{c}(z)$. The application of the constraints now leads to $\widehat{\mathrm{H}}_{c}(z)=\mathrm{H}_{c}^{o}(z)$ and hence $\widehat{h}=h^{o}$.

If we want the estimated channel to be reducible, then we have to apply the explicit constraint $h=T_{c}^{o} h_{I}$, which can be reformulated as the following implicit constraint: $\mathcal{K}_{\theta_{1}}=P_{T_{c}^{o}}^{\perp} h=0$. It turns out that in this case of deterministic input symbols, we can remain working in the complex domain, which we shall do. So we get $\frac{\partial \mathcal{K}_{\theta_{1}}^{H}}{\partial \theta_{1}}=P_{T_{c}^{o}}^{\perp}$ and we can take $\mathcal{V}_{\theta_{1}}=P_{T_{c}^{o}}$. Hence, the constrained CRB for $h$ satisfying the constraints $P_{T_{c}^{o}}^{\perp} h=0$ (compare to (32)) and $h^{o H} h=h^{o H} h^{o}$ is

$$
C R B_{C, h}=\left(P_{T_{c}} \mathcal{A}^{H} P_{\mathcal{T}(h)}^{\perp} \mathcal{A} P_{T_{c}}\right)^{+}=\left(P_{T_{c}} J_{h h}(\theta) P_{T_{c}}\right)^{+}
$$

which in general will be smaller than $J_{h h}^{+}(\theta)$ since more a priori information is introduced (in the form of $m\left(N_{c}-1\right)+1$ constraints, compared to the minimal number of $N_{c}$ constraints to ensure identifiability).

\subsection{Gaussian Model}

In the Gaussian model, the estimation of $h$ is not decoupled from the estimation of $\sigma_{v}^{2}$ and the estimation parameter is $\theta=\left[\begin{array}{ll}h^{H} & \sigma_{v}^{2}\end{array}\right]^{H}$. Unlike in the deterministic model, as $J_{\theta \theta^{*}} \neq 0$, we cannot treat the complex and real constellations together. Identifiability here occurs from $C_{Y Y}(\theta)$ as $m_{Y}(\theta) \equiv 0$. Blind identifiability in the Gaussian model does not require the channel to be irreducible [1].

\subsubsection{FIMs}

Circular Complex Symbol Constellation When the input constellation is complex, the FIM computation is based on the complex probability density function of $\boldsymbol{Y}$ :

$$
\boldsymbol{Y} \sim \mathcal{N}\left(m_{Y}, C_{Y Y}\right), \quad \text { with } \quad C_{Y Y}=\sigma_{a}^{2} \mathcal{T}(h) \mathcal{T}^{H}(h)+\sigma_{v}^{2} I, \quad m_{Y}=0 .
$$

Let $h_{R}=\left[\operatorname{Re}\left(h^{T}\right) \operatorname{Im}\left(h^{T}\right)\right]^{T}$ and $\bar{\theta}_{R}=\left[\begin{array}{ll}h_{R}^{T} & \sigma_{v}^{2}\end{array}\right]^{T}$, the real parameter vector. As $J_{\theta \theta^{*}}$ is non zero, we cannot consider the complex CRB anymore: the real $\operatorname{FIM} \mathcal{J}_{\bar{\theta}_{R} \bar{\theta}_{R}}$ is determined via 77 thanks to the quantities:

$$
J_{\theta \theta}(i, j)=\operatorname{tr}\left\{C_{Y Y}^{-1}\left(\frac{\partial C_{Y Y}}{\partial \theta_{i}^{*}}\right) C_{Y Y}^{-1}\left(\frac{\partial C_{Y Y}}{\partial \theta_{j}^{*}}\right)^{H}\right\}
$$




$$
\begin{gathered}
J_{\theta \theta^{*}}(i, j)=\operatorname{tr}\left\{C_{Y Y}^{-1}\left(\frac{\partial C_{Y Y}}{\partial \theta_{i}^{*}}\right) C_{Y Y}^{-1}\left(\frac{\partial C_{Y Y}}{\partial \theta_{j}^{*}}\right)\right\} \\
\text { where: }\left\{\begin{array}{l}
\frac{\partial C_{Y Y}}{\partial h_{i}^{*}}=\sigma_{a}^{2} \mathcal{T}(h) \mathcal{T}^{H}\left(\frac{\partial h}{\partial h_{i}^{*}}\right) \\
\frac{\partial C_{Y Y}}{\partial \sigma_{v}^{2}}=\frac{1}{2} I .
\end{array}\right.
\end{gathered}
$$

Real Symbol Constellation When the input constellation is real, the FIM is:

$$
\begin{aligned}
& \mathcal{J}_{\theta \theta}(i, j)=\frac{1}{2} \operatorname{tr}\left\{C_{Y Y}^{-1}\left(\frac{\partial C_{Y Y}}{\partial \theta_{i}}\right) C_{Y Y}^{-1}\left(\frac{\partial C_{Y Y}}{\partial \theta_{j}}\right)^{T}\right\} \\
& \left\{\begin{array}{l}
\frac{\partial C_{Y Y}}{\partial h_{i}}=\sigma_{a}^{2} \mathcal{T}(h) \mathcal{T}^{T}\left(\frac{\partial h}{\partial h_{i}}\right)+\sigma_{a}^{2} \mathcal{T}\left(\frac{\partial h}{\partial h_{i}}\right) \mathcal{T}^{T}(h) \\
\frac{\partial C_{Y Y}}{\partial \sigma_{v}^{2}}=I .
\end{array}\right.
\end{aligned}
$$

\subsubsection{FIM singularities}

Circular Complex Symbol Constellation Under the Gaussian blind identifiability conditions [1, a complex channel $h$ is identifiable up to a phase factor. This corresponds to one singularity of the global FIM $\mathcal{J}_{\bar{\theta}_{R} \bar{\theta}_{R}}$ :

$$
\mathcal{J}_{\bar{\theta}_{R} \bar{\theta}_{R}}=\left[\begin{array}{cc}
\mathcal{J}_{h_{R} h_{R}} & \mathcal{J}_{h_{R} \sigma_{v}^{2}} \\
\mathcal{J}_{\sigma_{v}^{2} h_{R}} & \mathcal{J}_{\sigma_{v}^{2} \sigma_{v}^{2}}
\end{array}\right]
$$

as well as of:

$$
\mathcal{J}_{h_{R} h_{R}}\left(\bar{\theta}_{R}\right)=\mathcal{J}_{h_{R} h_{R}}-\mathcal{J}_{h_{R} \sigma_{v}^{2}}\left(\mathcal{J}_{\sigma_{v}^{2} \sigma_{v}^{2}}\right)^{-1} \mathcal{J}_{\sigma_{v}^{2} h_{R}}
$$

$\mathcal{J}_{h_{R} h_{R}}^{-1}\left(\bar{\theta}_{R}\right)$ would be the unconstrained $\mathrm{CRB}$ for $h$ if its estimation were regular. The null space of $\mathcal{J}_{h_{R} h_{R}}\left(\bar{\theta}_{R}\right)$ is spanned by

$$
h_{S}=\left[-\operatorname{Im}^{T}(h) \operatorname{Re}^{T}(h)\right]^{T}=h_{S_{2}} .
$$

Real Symbol Constellation The real FIM $\mathcal{J}_{\theta \theta}$ is regular under the identifiability conditions, as well as $\mathcal{J}_{h h}(\theta)$.

\subsubsection{Equivalence between FIM regularity and local identifiability}

Theorem 14 The (complex or real) FIM is singular if and only if there exist a vector $h^{\prime}$ (complex or real) and a scalar $\sigma_{v}^{2}$ such that:

$$
\sigma_{a}^{2} \mathcal{T}(h) \mathcal{T}^{H}\left(h^{\prime}\right)+\sigma_{a}^{2} \mathcal{T}\left(h^{\prime}\right) \mathcal{T}^{H}(h)+\sigma_{v}^{2^{\prime}} I=0
$$

Proof:

Complex case: The complex FIM matrix is singular if there exists a $\bar{\theta}_{R}^{\prime}=\left[\operatorname{Re}^{T}\left(h^{\prime}\right) \operatorname{Im}^{T}\left(h^{\prime}\right) \sigma_{v}^{2}\right]^{T}$, such that:

$$
\begin{gathered}
\mathcal{J}_{\bar{\theta}_{R} \bar{\theta}_{R} \bar{\theta}_{R}^{\prime}=0} \Leftrightarrow\left[\left(\frac{\partial \operatorname{vec}^{T}\left\{C_{Y Y}\right\}}{\partial h^{*}}\right)^{T}\left(\frac{\partial \operatorname{vec}^{T}\left\{C_{Y Y}\right\}}{\partial h}\right)^{T}\left(\frac{\partial \operatorname{vec}^{T}\left\{C_{Y Y}\right\}}{\partial \sigma_{v}^{2}}\right)^{T}\right]\left[\begin{array}{c}
h^{\prime} \\
h^{\prime *} \\
\sigma_{v}^{2}
\end{array}\right]=0 \\
\Leftrightarrow \sum_{j}\left(\frac{\partial C_{Y Y}}{\partial h_{j}^{*}}\right)^{H} h_{j}^{\prime}+\sum_{j}\left(\frac{\partial C_{Y Y}}{\partial h_{j}^{*}}\right) h_{j}^{\prime *}+\frac{1}{2}{\sigma_{v}^{2}}^{\prime} I=0 .
\end{gathered}
$$


We have: $\frac{\partial C_{Y Y}}{\partial h_{j}^{*}}=\sigma_{a}^{2} \mathcal{T}(h) \mathcal{T}^{H}\left(\frac{\partial h}{\partial h_{i}}\right)$ and $\sum_{j} \mathcal{T}\left(\frac{\partial h}{\partial h_{i}}\right) h_{j}^{\prime}=\mathcal{T}\left(h^{\prime}\right)$, then:

$$
79 \Leftrightarrow \sigma_{a}^{2} \mathcal{T}\left(h^{\prime}\right) \mathcal{T}^{H}(h)+\sigma_{a}^{2} \mathcal{T}(h) \mathcal{T}^{H}\left(h^{\prime}\right)+\frac{1}{2} \sigma_{v}^{2^{\prime}} I=0 .
$$

which is equivalent to equation $(78)$ (with $\frac{1}{2} \sigma_{v}^{2 \prime} \rightarrow \sigma_{v}^{2 \prime}$ ).

Real case: The real FIM matrix is singular if there exists a $\theta^{\prime}=\left[\begin{array}{ll}h^{\prime T} & \sigma_{v}^{2^{\prime}}\end{array}\right]^{T}$, such that:

$$
\begin{gathered}
\mathcal{J}_{\theta \theta} \theta^{\prime}=0 \\
\Leftrightarrow\left[\left(\frac{\partial \operatorname{vec}^{T}\left\{C_{Y Y}\right\}}{\partial h}\right)^{H}\left(\frac{\partial \operatorname{vec}^{T}\left\{C_{Y Y}\right\}}{\partial \sigma_{v}^{2}}\right)^{H}\right]^{H}\left[\begin{array}{c}
h^{\prime} \\
\sigma_{v}^{2}{ }^{\prime}
\end{array}\right]=0 \\
\Leftrightarrow \sum_{j}\left(\frac{\partial C_{Y Y}}{\partial h_{j}}\right)^{H} h_{j}^{\prime}+\sigma_{v}^{2 \prime} I=0 .
\end{gathered}
$$

We have $\frac{\partial C_{Y Y}}{\partial h_{j}}=\sigma_{a}^{2} \mathcal{T}(h) \mathcal{T}^{H}\left(\frac{\partial h}{\partial h_{i}}\right)+\sigma_{a}^{2} \mathcal{T}^{H}\left(\frac{\partial h}{\partial h_{i}}\right) \mathcal{T}^{H}(h)$. Then:

$$
83 \Leftrightarrow \sigma_{a}^{2} \mathcal{T}\left(h^{\prime}\right) \mathcal{T}^{H}(h)+\sigma_{a}^{2} \mathcal{T}(h) \mathcal{T}^{H}\left(h^{\prime}\right)+\sigma_{v}^{2 \prime} I=0 .
$$

From $(78)$, we can deduce the following theorem.

Theorem 15 The real/complex channel is locally blindly identifiable if and only if the FIM is regular/1singular.

Note that locally a complex channel is identifiable up to a continuous phase factor but a real channel is locally identifiable strictly speaking.

Proof: Assume that the FIM has a null vector $\theta^{\prime}=\left[\begin{array}{ll}h^{\prime T} & \sigma_{v}^{2}\end{array}\right]^{T}$ which in the complex channel case is non colinear to $h_{S}$. Then theorem 14 says that $\theta^{\prime}$ satisfy (78). Now, with $\epsilon>0$ arbitrarily small,

$$
\begin{aligned}
C_{Y Y}\left(\theta+\epsilon \theta^{\prime}\right)-C_{Y Y}(\theta) & =\left(\sigma_{a}^{2} \mathcal{T}\left(h+\epsilon h^{\prime}\right) \mathcal{T}^{H}\left(h+\epsilon h^{\prime}\right)+\left(\sigma_{v}^{2}+\epsilon \sigma_{v}^{2}{ }^{\prime}\right) I\right)-\left(\sigma_{a}^{2} \mathcal{T}(h) \mathcal{T}^{H}(h)+\sigma_{v}^{2} I\right) \\
& =\sigma_{a}^{2} \mathcal{T}(h) \mathcal{T}^{H}\left(\epsilon h^{\prime}\right)+\sigma_{a}^{2} \mathcal{T}\left(\epsilon h^{\prime}\right) \mathcal{T}^{H}(h)+\epsilon \sigma_{v}^{\prime \prime} I+O\left(\epsilon^{2}\right)=O\left(\epsilon^{2}\right) .
\end{aligned}
$$

This means that the covariance matrix is locally constant in the direction of $\theta^{\prime}$ around $\theta$. Similarly to the proof of theorem 12 one can show that if the channel is identifiable, the FIM is regular or 1-singular.

In Appendix B, we study the conditions on the characteristics of the channel to have local identifiability. The results are contained in the theorem below. The channel is assumed reducible: $\mathbf{H}(z)=$ $\mathbf{H}_{I}(z) \mathrm{H}(z)$.

Theorem 16 The Gaussian FIM for a real/complex multichannel is regular/1-singular and the channel is locally blindly identifiable if:

(1) $M \geq \max \left(\underline{M}_{I}+1, N_{c}-1\right)$,

(2) the channel has no conjugate reciprocal zeros, i.e. there exists no $z_{o} \in \mathbb{R} / \mathbb{C}$ such that $\mathbf{H}\left(z_{o}\right)=$ $\mathbf{H}\left(1 / z_{o}^{*}\right)=0$.

Proof: Appendix B

The no conjugate reciprocal zeros condition was also given in [17, but for the real channel case only, without mentioning that the complex case is singular in any case. Remark that, in particular, the Gaussian FIM is regular if there are arbitrary zeros (not in conjugate reciprocal pairs) due to the fact that a minimum phase channel is identifiable (example of local identifiability).

The monochannel case is treated in Appendix A the results mentioned above for a multichannel are valid here also except that the noise variance $\sigma_{v}^{2}$ cannot be identified, which results in an additional singularity of the FIM when the channel has no conjugate reciprocal zeros (when the channel has conjugate reciprocal zeros, there is no additional singularity). 


\subsubsection{Regularized Blind CRBs}

Complex symbol constellation As in the deterministic case, we need to define a regularized CRB, by introducing some a priori knowledge on the parameters, allowing us to determine the ambiguous phase factor. We assume that the channel is (blindly) identifiable: we do not treat the monochannel or conjugate reciprocal zeros.

The estimation of $h_{R}$ is considered under the constraint:

$$
h_{S_{2}}^{o T} h_{R}=0
$$

which leads to the constrained $\mathrm{CRB}$ for $h_{R}$ :

$$
C R B_{C, h_{R}}=\mathcal{J}_{h_{R} h_{R}}^{+}(\theta) .
$$

This linear constraint does not allow to estimate the phase factor completely and a sign ambiguity is left but not reflected in the FIM singularities as it is a discrete ambiguity. For MSE computation purposes, the sign ambiguity can be resolved by requiring $h_{R}^{o T} h_{R}>0$, which together with 88 can be stated as $h^{o H} h>0$.

Real symbol constellation No regularization is necessary and the CRB is $\mathcal{J}_{h h}^{-1}(\theta)$. To compare the MSE for an estimator to this CRB, the knowledge of the right sign and right phase of the zeros (e.g. minimum phase in the reducible case) should be used.

\section{Conclusions}

We have provided a compact derivation of the CRB under parameter constraints. In blind channel estimation under the deterministic or Gaussian symbol model, the estimation problem has indeed to be augmented with constraints to remove singularities. We have introduced the notion of minimal constraints and shown how several intuitively attractive and hence popular constraint sets lead simply to the pseudo inverse of the FIM as constrained CRB. For the blind channel estimation problem, we have illustrated the connection between local identifiability problems and FIM singularities. In fact, if the symbol model would be a discrete alphabet constellation [18, no more continuous ambiguities would persist and the unconstrained CRB would typically exist. Deterministic and Gaussian symbol models have their raison d'être though, since they are typically less plagued with local minima problems and hence can lead to estimates with which to initialize methods based on discrete symbol alphabets.

\section{Appendix A}

For a minimal number of independent constraints, we prove that $C R B_{C}=\mathcal{J}_{\theta \theta}^{+}$is the constrained CRB which gives the lowest value for $\operatorname{tr}\left\{C R B_{C}\right\}$ and is attained when range $\left\{\frac{\partial \mathcal{K}_{\theta}}{\partial \theta}\right\}=\operatorname{null}\left(\mathcal{J}_{\theta \theta}\right)$.

Let $\mathcal{K}_{\theta}=0$ be an arbitrary set of constraints on $\theta ; \mathcal{V}_{\theta}=\left(\frac{\partial \mathcal{K}_{\theta}^{T}}{\partial \theta}\right)^{\perp}$ has full column rank and we assume that $\mathcal{V}_{\theta}^{T} \mathcal{J}_{\theta \theta} \mathcal{V}_{\theta}$ is invertible. The corresponding constrained CRB is:

$$
C R B_{C}=\mathcal{V}_{\theta}\left(\mathcal{V}_{\theta}^{T} \mathcal{J}_{\theta \theta} \mathcal{V}_{\theta}\right)^{-1} \mathcal{V}_{\theta}^{T}
$$

Introduce the eigendecomposition of $\mathcal{J}_{\theta \theta}=S_{1} \Lambda_{1} S_{1}^{T}+S_{2} 0 S_{2}^{T}$. In general, $\mathcal{V}_{\theta}$ has components along $S_{1}$ and $S_{2}: \mathcal{V}_{\theta}=S_{1} Q_{1}+S_{2} Q_{2}$. The fact that the constraints $\mathcal{K}_{\theta}$ are independent and minimal in number implies that $Q_{1}$ is square and invertible. Then we obtain

$$
\begin{aligned}
C R B_{C} & =\mathcal{V}_{\theta}\left(\mathcal{V}_{\theta}^{T} S_{1} \Lambda_{1} S_{1}^{T} \mathcal{V}_{\theta}\right)^{-1} \mathcal{V}_{\theta}^{T} \\
& =\mathcal{V}_{\theta}\left(Q_{1}^{T} \Lambda_{1} Q_{1}\right)^{-1} \mathcal{V}_{\theta}^{T} \\
& =\mathcal{V}_{\theta} Q_{1}^{-1} \Lambda_{1}^{-1} Q_{1}^{-T} \mathcal{V}_{\theta}^{T} \\
& =\left(S_{1}+S_{2} Q_{2} Q_{1}^{-1}\right) \Lambda_{1}^{-1}\left(S_{1}+S_{2} Q_{2} Q_{1}^{-1}\right)^{T}
\end{aligned}
$$

The difference between the $C R B_{C}$ and $\mathcal{J}_{\theta \theta}^{+}=S_{1} \Lambda_{1}^{-1} S_{1}{ }^{H}$ may be indefinite in general, however:

$$
\operatorname{tr}\left(C R B_{C}\right)=\operatorname{tr}\left(\mathcal{J}_{\theta \theta}^{+}\right)+\operatorname{tr}\left(Q_{2} Q_{1}^{-1} \Lambda_{1}^{-1} Q_{1}^{-T} Q_{2}^{T}\right)
$$


The second term is nonnegative, so $\operatorname{tr}\left(C R B_{C}\right) \geq \operatorname{tr}\left(\mathcal{J}_{\theta \theta}^{+}\right)$, with equality iff $Q_{2}=0$, i.e. range $\left\{\frac{\partial \mathcal{K}_{\theta}^{T}}{\partial \theta}\right\}=$ $\operatorname{null}\left(\mathcal{J}_{\theta \theta}\right)$ or range $\left\{\mathcal{V}_{\theta}\right\}=$ range $\left\{\mathcal{J}_{\theta \theta}\right\}$. In that case $C R B_{C}=\mathcal{J}_{\theta \theta}^{+}$.

\section{Appendix B}

In this appendix, we study the solutions $\left(h^{\prime}, \sigma_{v}^{2^{\prime}}\right)$ of the equation:

$$
\mathcal{T}(h) \mathcal{T}^{H}\left(h^{\prime}\right)+\mathcal{T}\left(h^{\prime}\right) \mathcal{T}^{H}(h)+\sigma_{v}^{2^{\prime}} I=0 .
$$

We first treat the monochannel case for a complex or real channel, which allows us to then treat the multichannel case.

\section{B.1 Complex Monochannel}

We assume that $M \geq N-1$; in this case, equation 93 can be written in the $z$-domain as:

$$
\sigma_{a}^{2} \mathrm{H}(z) \mathrm{H}^{\prime \dagger}(z)+\sigma_{a}^{2} \mathrm{H}^{\prime}(z) \mathrm{H}^{\dagger}(z)+\sigma_{v}^{2 \prime}=0
$$

where $\mathrm{H}^{\dagger}(z)=\mathrm{H}^{H}\left(1 / z^{*}\right)$. Let's denote $p(z)=\mathrm{H}(z) \mathrm{H}^{\prime \dagger}(z)$, then:

$$
94 \Leftrightarrow p(z)+p^{\dagger}(z)+\sigma_{v}^{2^{\prime}}=0 \text {. }
$$

B.1.1 Solutions of the form $\left[\begin{array}{lll}* & * & 0\end{array}\right]^{T}:{\sigma_{v}^{2}}^{\prime}=0$.

$$
\begin{gathered}
p(z)=\sum_{i=-d_{p}}^{d_{p}^{\prime}} \alpha_{i} z^{-i} \text { and } p^{\dagger}(z)=\sum_{i=-d_{p}^{\prime}}^{d_{p}} \alpha_{-i}^{*} z^{-i} \\
p(z)+p^{\dagger}(z)=0 \Rightarrow d_{p}^{\prime}=d_{p} \quad\left(\text { and } \alpha_{i}=-\alpha_{-i}^{*}\right) .
\end{gathered}
$$

As $\mathrm{H}(z)$ and $\mathrm{H}^{\dagger}(z)$ are respectively causal and anticausal, $\operatorname{deg}(\mathrm{H}(z))=\operatorname{deg}\left(\mathrm{H}^{\prime}(z)\right)=N-1=d_{p}$. In the following, we assume that $\mathrm{H}(z)$ is monic. Equation (95) is also equivalent to:

$$
p(z)=-p^{\dagger}(z) \text {. }
$$

From this equation, we can deduce that if $z_{o}$ is a zero of $p(z)$, so is $1 / z_{o}^{*}$, which implies that $p(z)$ is of the form:

$$
p(z)=\alpha \prod_{i=0}^{N_{1}-1}\left(1-z_{i} z^{-1}\right)\left(1-z_{i}^{*} z\right)\left[\left(1-z^{-1}\right)(1+z)\right]^{N_{2}} .
$$

where $N_{1}+N_{2}=N-1$. We will differentiate the zeros that are equal to 1 or $-1:\left\{z_{i}\right\}_{i=1: N_{1}-1}$ are different from 1 or -1 . $z$ equals 1 or -1 .

The number of singularities depends on the characteristics of the channel $\mathrm{H}(z)$ and namely the presence of conjugate reciprocal zeros.

(1) $\mathrm{H}(z)$ has no conjugate reciprocal zeros:

The $N-1$ zeros of $\mathrm{H}(z)$ are among the zeros of $p(z)$, this implies that $p(z)$ has no zeros equal to 1 or -1 :

$$
p(z)=\alpha \prod_{i=0}^{N-2}\left(1-z_{i} z^{-1}\right) \prod_{i=0}^{N-2}\left(1-z_{i}^{*} z\right)=\mathrm{H}(z) \mathrm{H}^{\prime \dagger}(z)
$$

furthermore, without loss of generality, we can assume that:

$$
\mathrm{H}(z)=\prod_{i=0}^{N-2}\left(1-z_{i} z^{-1}\right) .
$$

In that case:

$$
\begin{gathered}
\mathrm{H}^{\prime \dagger}(z)=\alpha \prod_{i=0}^{N-1}\left(1-z_{i}^{*} z\right)=\alpha \mathrm{H}^{\dagger}(z) \\
95 \Rightarrow \mathrm{H}^{\prime}(z)=j \mathrm{H}(z) .
\end{gathered}
$$

The FIM is 1-singular. Its null space is spanned by $\left[-\operatorname{Im}^{T}(h) \operatorname{Re}^{T}(h)\right]^{T}$. 
(2) $\mathrm{H}(z)$ has 1 pair of conjugate reciprocal zeros: $\left(z_{o}, 1 / z_{o}^{*}\right), z_{o} \neq 1, z_{o} \neq-1$.

Again, without loss of generality, we can assume that:

$$
\mathrm{H}(z)=\left(1-z_{o} z^{-1}\right)\left(1-z_{o}^{*} z\right) z^{-1} z_{o}^{-*} \underbrace{\prod_{i=1}^{N-3}\left(1-z_{i} z^{-1}\right)}_{\mathrm{H}_{1}(z)} .
$$

There are 2 degrees of freedom in $\mathrm{H}^{\prime}(z)$ coming from the fact that $\mathrm{H}^{\prime}(z)$ can admit 1 and -1 as zeros or not. Two possible choices for $\mathrm{H}^{\prime}(z)$ are then:

$$
\left\{\begin{array}{l}
\mathrm{H}^{\prime}(z)=j\left(1-z_{N-1} z^{-1}\right)\left(z^{-1}-z_{N-1}^{*}\right) z_{o} \mathrm{H}_{1}(z) \\
\mathrm{H}^{\prime}(z)=\left(1-z^{-1}\right)\left(z^{-1}-1\right) z_{o} \mathrm{H}_{1}(z)
\end{array}\right.
$$

The FIM has 2 singularities coming from the pair of conjugate reciprocal zeros, and 1 singularity corresponding to $j \mathrm{H}(z)$.

(3) $\mathrm{H}(z)$ has one zero equal to 1 or -1 .

We assume that this zero is equal $1 . \mathrm{H}^{\prime}(z)$ can be chosen as:

$$
\mathrm{H}(z)=\left(1-z^{-1}\right) \underbrace{\prod_{i=0}^{N-3}\left(1-z_{i} z^{-1}\right)}_{\mathrm{H}_{1}(z)}, \mathrm{H}^{\prime}(z)=\left(1+z^{-1}\right) \mathrm{H}_{1}(z) \text {. }
$$

(4) $\mathrm{H}(z)$ has several conjugate reciprocal zeros:

Then, to each pair of conjugate reciprocal zeros different from 1 and -1 , correspond 2 singularities, and to each zero equal to 1 or -1 corresponds 1 singularity.

\section{B.1.2 Solutions of the form $\left[\begin{array}{lll}* & * & \sigma_{v}^{2}\end{array}\right]^{T}: \sigma_{v}^{2^{\prime}} \neq 0$}

(1) $\mathrm{H}(z)$ admits conjugate reciprocal zeros $z_{o}$ :

$$
\underbrace{p\left(z_{o}\right)}_{=0}+\underbrace{p^{\dagger}\left(z_{o}\right)}_{=0}+\sigma_{v}^{2^{\prime}}=0 \Rightarrow \sigma_{v}^{2^{\prime}}=0
$$

So there is no singular vector of the desired form in this case.

(2) $\mathrm{H}(z)$ has no conjugate reciprocal zeros:

$\mathrm{H}(z)$ is of the form $\mathrm{H}(z)=\prod_{i=0}^{N-2}\left(1-z_{i} z^{-1}\right)$. One can verify that

$$
\mathrm{H}^{\prime}(z)=\prod_{i=0}^{N-2}\left(1+z_{i} z^{-1}\right)
$$

is such that:

$$
\sigma_{a}^{2} \mathrm{H}(z) \mathrm{H}^{\prime}(z)+\sigma_{a}^{2} \mathrm{H}^{\prime}(z) \mathrm{H}(z)=\sigma_{a}^{2} 2^{N-1} \prod_{i=0}^{N-2}\left(1-\left\|z_{i}\right\|^{2}\right)
$$

And so $H^{\prime}(z)$ and $\sigma_{v}^{2}{ }^{\prime}=-\sigma_{a}^{2} 2^{N-1} \prod_{i=0}^{N-2}\left(1-\left\|z_{i}\right\|^{2}\right)$ verify 94 ; and it can also be proved that this is the only singular vector due to the unidentifiability of $\sigma_{v}^{2}$. It can also be verified that $\mathrm{H}^{\prime}(z)$ is not a solution of (94) if $\mathrm{H}(z)$ has conjugate reciprocal zeros. 


\section{B.2 Real Monochannel}

B.2.1 Solutions of the from $\left[\begin{array}{lll}* & * & 0\end{array}\right]^{T}:{\sigma_{v}^{2}}^{\prime}=0$.

Similar reasonings apply here.

(1) $\mathrm{H}(z)$ has no pair of conjugate reciprocal zeros:

$p(z)+p^{\dagger}(z)=0$ can only be satisfied by $p(z) \equiv 0$. So the FIM is regular.

(2) $\mathrm{H}(z)$ has 1 pair conjugate reciprocal zeros: $\left(z_{o}, 1 / z_{o}^{*}\right), z_{o} \neq 1, z_{o} \neq-1$.

$$
\mathrm{H}(z)=\left(1-z_{o} z^{-1}\right)\left(1-z_{o} z\right) z^{-1} z_{o}^{-1} \underbrace{\prod_{i=1}^{N-3}\left(1-z_{i} z^{-1}\right)\left(1-z_{i} z\right)}_{\mathrm{H}_{1}(z)} .
$$

There is now only $1 \mathrm{H}^{\prime}(z)$ possible (the first solution in 105 is not valid here):

$$
\mathrm{H}^{\prime}(z)=\left(1-z^{-1}\right)(1-z) z^{-1} z_{o} \mathrm{H}_{1}(z) .
$$

The FIM has 1 singularity.

(3) $\mathrm{H}(z)$ has one zero equal to 1 or -1 .

We assume that this zero is $1 . \mathrm{H}^{\prime}(z)$ can be chosen as:

$$
\mathrm{H}(z)=\left(1-z^{-1}\right) \underbrace{\prod_{i=0}^{N-2}\left(1-z_{i} z^{-1}\right)}_{\mathrm{H}_{1}(z)}, \mathrm{H}^{\prime}(z)=\left(1+z^{-1}\right) \mathrm{H}_{1}(z) .
$$

(4) $\mathrm{H}(z)$ has several conjugate reciprocal zeros:

Then, to each pair of conjugate reciprocal zeros different from 1 and -1 , and to each zero equal to 1 or -1 corresponds 1 singularity.

\section{B.2.2 Solutions of the form $\left[\begin{array}{lll}* & * & \sigma_{v}^{2}\end{array}\right]^{H}: \sigma_{v}^{2^{\prime}} \neq 0$}

The same singularity as in the complex case, due to the inidentifiability of $\sigma_{v}^{2}$, appears (except again if the channel $\mathrm{H}(z)$ has conjugate reciprocal zeros).

\section{B.3 Multichannel}

Assume now that $\mathbf{H}(z)$ is a true multichannel, possibly reducible:

$$
\mathbf{H}(z)=\mathbf{H}_{I}(z) \mathrm{H}_{c}(z) .
$$

As for the monochannel case, we search first the solutions of the form: $\left[\operatorname{Re}^{T}\left(h^{\prime}\right) \operatorname{Im}^{T}\left(h^{\prime}\right) \quad 0\right]^{T}$. Then $h^{\prime}$ verifies:

$$
\mathcal{T}(h) \mathcal{T}^{H}\left(h^{\prime}\right)+\mathcal{T}\left(h^{\prime}\right) \mathcal{T}^{H}(h)=0 .
$$

The burst length is assumed to be $M \geq \underline{M}+1$ which can be lower than $N-1$ (so the transposition to the z-domain is not as convenient as in the monochannel case). The previous equation implies that $\mathcal{T}\left(h^{\prime}\right)$ should have for effect to reduce the previous quantity to at least the same rank as $\mathcal{T}(h)$. So:

$$
\text { range }\left\{\mathcal{T}\left(h^{\prime}\right)\right\} \subset \text { range }\{\mathcal{T}(h)\}
$$

which implies (see e.g. Appendix B in [1]):

$$
\begin{aligned}
& \mathbf{H}^{\prime}(z)=\mathbf{H}_{I}(z) \mathrm{H}_{c}^{\prime}(z) \\
& \left\{\begin{array}{rl}
\mathbf{H}^{\prime}(z) & =\mathbf{H}_{I}(z) \mathrm{H}_{c}^{\prime}(z) \\
\mathbf{H}(z) & =\mathbf{H}_{I}(z) \mathrm{H}_{c}(z)
\end{array} \Rightarrow \mathcal{T}\left(h_{I}\right) \mathcal{T}\left(h_{c}\right) \mathcal{T}^{H}\left(h_{c}^{\prime}\right) \mathcal{T}^{H}\left(h_{I}\right)+\mathcal{T}\left(h_{I}\right) \mathcal{T}\left(h_{c}^{\prime}\right) \mathcal{T}^{H}\left(h_{c}\right) \mathcal{T}^{H}\left(h_{I}\right)=0 .\right.
\end{aligned}
$$


As $\mathcal{T}\left(h_{I}\right)$ is full column-rank,

$$
117 \Leftrightarrow \mathcal{T}\left(h_{c}\right) \mathcal{T}^{H}\left(h_{c}^{\prime}\right)+\mathcal{T}\left(h_{c}^{\prime}\right) \mathcal{T}^{H}\left(h_{c}\right)=0 .
$$

As $\mathcal{T}\left(h_{c}\right)$ is of length at least $N_{c}-1$ according to the identifiability conditions, (118) implies:

$$
\mathrm{H}_{c}(z) \mathrm{H}_{c}^{\prime \dagger}(z)+\mathrm{H}_{c}^{\prime \dagger}(z) \mathrm{H}_{c}(z)=0
$$

which leads to the monochannel case treated previously.

As for the solutions of the form $\left[* \sigma_{v}^{2^{\prime}}\right]^{T}, \sigma_{v}^{2^{\prime}} \neq 0$, there are none in this case ( $\sigma_{v}^{2}$ is identifiable in any case).

\section{References}

[1] E. de Carvalho and D.T.M. Slock, "Blind and Semi-Blind FIR Multichannel Estimation: Identifiability Conditions," Submitted to IEEE Transactions on Signal Processing.

[2] E. Moulines, P. Duhamel, J.F. Cardoso, and S. Mayrargue, "Subspace Methods for the Blind Identification of Multichannel FIR filters," IEEE Transactions on Signal Processing, vol. 43, no. 2, pp. 516-526, Feb. 1995.

[3] E. de Carvalho and D.T.M. Slock, "Blind and Semi-Blind FIR Multichannel Estimation: CramérRao Bounds," Submitted to IEEE Transactions on Signal Processing.

[4] J.D. Gorman and A.O. Hero, "Lower Bounds for Parametric Estimation with Constraints," IEEE Transactions on Information Theory, vol. 26, no. 6, pp. 1285-1301, Nov. 1990.

[5] K. Abed Meraim, E. Moulines, and P. Loubaton, "Prediction Error Method for Second-Order Blind Identification," IEEE Transactions on Signal Processing, vol. 45, no. 3, pp. 694-705, March 1997.

[6] G.B. Giannakis and S.D. Halford, "Asymptotically Optimal Blind Fractionally-Spaced Channel Estimation and Performance Analysis," IEEE Transactions on Signal Processing, vol. 45, no. 7, pp. 1815-1830, July 1997.

[7] S.M. Kay, Fundamentals of Statistical Signal Processing Estimation Theory, Prentice-Hall, Englewood Cliffs, NJ, 1993.

[8] Peter E. Caines, Linear Stochastic Systems, John Wiley \& Sons, 1988.

[9] B. Hochwald and A. Nehorai, "On Identifiability and Information-Regularity in Parameterized Normal Distributions," Circuits, Systems ans Signal Processing, vol. 16, no. 1, 1997.

[10] T. Marzetta, "On Simple Derivation of the Constrained Multiple Parameter Cramér-Rao Bound," IEEE Transactions on Signal Processing, vol. 41, no. 6, pp. 2247-2249, June 1993.

[11] P. Stoica and B.C. Ng, "On the Cramér-Rao Bound Under Parametric Constraints," IEEE Signal Processing Letters, vol. 5, no. 7, pp. 177-179, July 1998.

[12] E. de Carvalho and D.T.M. Slock, "Cramér-Rao Bounds for Semi-blind, Blind and Training Sequence based Channel Estimation," in Proc. SPAWC 97 Conf., Paris, France, April 1997.

[13] Y. Hua, "Fast Maximum Likelihood for Blind Identification of Multiple FIR Channels," IEEE Transactions on Signal Processing, vol. 44, no. 3, pp. 661-672, March 1996.

[14] Y. Hua and M. Wax, "Strict Identifiability of Multiple FIR Channels Driven by an Unknown Arbitrary Sequence," IEEE Transactions on Signal Processing, vol. 44, no. 3, pp. 756-759, March 1996.

[15] J.L. Bapat, Partially Blind Identification of FIR Channels for QAM Signals, Ph.D. thesis, Pennsylvania State University, Aug. 1996.

[16] D.R. Morgan, J. Benesty, and M.M. Sondhi, "On the Evaluation of Estimated Impulse Responses," IEEE Signal Processing Letters, vol. 5, no. 7, pp. 174-176, July 1998.

[17] H.H. Zeng and L. Tong, "Blind Channel Estimation Using the Second-Order Statistics: Asymptotic Performance and Limitations," IEEE Transactions on Signal Processing, vol. 45, no. 8, pp. 20602071, Aug. 1997.

[18] H.A. Cirpan and M.K. Tsatsanis, "Stochatic Maximum-Likelihood Methods for Semi-Blind Channel Estimation," IEEE Signal Processing Letters, vol. 5, no. 1, pp. 21-24, Jan. 1998. 